\title{
THE SCHWARTZ ALGEBRA OF AN AFFINE HECKE ALGEBRA
}

\author{
PATRICK DELORME AND ERIC M. OPDAM
}

Date: November 5, 2018.

2000 Mathematics Subject Classification. Primary 20C08; Secondary 22D25, 22E35, 43A30.

During the preparation of this paper the second named author was partially supported by a Pionier grant of the Netherlands Organization for Scientific Research (NWO). 


\section{Contents}

1. Introduction 2

2. The affine Hecke algebra and the Schwartz algebra 5

2.1. The root datum and the affine Wevl group 5

2.2. Standard parabolic subsvstems 6

2.3. Label functions and root labels 6

$\begin{array}{ll}\text { 2.4. The Iwahori-Hecke algebra } & 7\end{array}$

2.5. Intertwining elements 9

2.6. Hilbert algebra structure on $\mathcal{H} \quad 10$

2.7. Discrete series representations 10

2.8. The Schwartz algebra: tempered representations 11

2.9. Casselman's criteria for temperedness 12

2.10. Exponents of finite functionals 12

2.11. The space $\mathbb{A}^{\text {temp }}$ of tempered finite functionals 13

2.12. Formal completion of $\mathcal{H}$ and Lusztig's structure theorem 14

3. Fourier Transform 16

3.1. Induction from standard parabolic subquotient algebras 16

$\begin{array}{lll}\text { 3.2. Groupoid of standard induction data } & 17\end{array}$

3.3. Fourier transform on $L_{2}(\mathcal{H}) \quad 20$

4. Main Theorem and its applications 22

4.1. Applications of the Main Theorem 23

5. Constant terms of matrix coefficients of $\pi(\xi)$

5.1. Definition of the constant terms of $f \in \mathbb{A}^{\text {temp }} \quad 25$

5.2. Constant terms of coefficients of $\pi(\xi)$ for $\xi \in \Xi_{\mu}$ generid 26

5.3. Some results for Wevl groups 28

5.4. The singularities of $f^{d} \quad 30$

6. Proof of the main theorem 34

6.1. Uniform estimates for the coefficients of $\pi(\xi) \quad 34$

6.2. Uniform estimates of the difference of a coefficient and its constant term 37

6.3. Wave packets 39

6.4. End of the proof of the main Theorem 41

7. Appendix: Some applications of spectral projections 43

8. Appendix: The c-function 45

$\begin{array}{ll}\text { References } & 48\end{array}$

Index 49

\section{Introduction}

An affine Hecke algebra is associated to a root datum (with basis) $\mathcal{R}=\left(X, Y, R_{0}, R_{0}^{\vee}, F_{0}\right)$, where $X, Y$ are lattices with a perfect pairing, 
$R_{0} \subset X$ is a reduced root system, $R_{0}^{\vee} \subset Y$ is the coroot system and $F_{0}$ is a basis of $R_{0}$, together with a length multiplicative function $q$ of the affine Weyl group associated to $\mathcal{R}$. It is denoted by $\mathcal{H}(\mathcal{R}, q)$ or simply $\mathcal{H}$. It admits a natural prehibertian structure (provided $q$ has values in $\mathbb{R}_{+}$, which we assume throughout), and it acts on its completion $L_{2}(\mathcal{H})$ through bounded operators. Thus $\mathcal{H}$ is a Hilbert algebra in the sense of $[6]$.

The spectral decomposition of the left and right representation of this Hibert algebra has been made explicit by one of the authors (E.O., 14). We will denote by $\mathcal{F}$ the isomorphism between $L_{2}(\mathcal{H})$ and its decomposition into irreducible representations. We will call this map $\mathcal{F}$ the Fourier transform.

Another interesting completion of $\mathcal{H}$ is the Schwartz algebra $\mathcal{S} \subset$ $L_{2}(\mathcal{H})$, which is a Fréchet algebra completion of $\mathcal{H}[14$. The main theorem of this article is the characterisation of the image of $\mathcal{S}$ by the Fourier transform $\mathcal{F}$. This characterisation has several important consequences which are described in Section 4, Let us briefly discuss these applications.

First of all, we obtain the analog of Harish-Chandra's completeness Theorem for generalized principal series of real reductive groups. The representations involved in the spectral decomposition of $L_{2}(\mathcal{H})$ are, as representations of $\mathcal{H}$, subrepresentation of certain finite dimensional induced representations from parabolic subalgebras (which are subalgebras of $\mathcal{H}$ which themselves belong to the class of affine Hecke algebras). We call these the standard tempered induced representations. There exist standard interwining operators (see 14]) between the standard induced tempered representations. The completeness Theorem states that the commutant of the standard tempered induced representations is generated by the self-intertwining operators given by standard intertwining operators.

Next we determine the image of the center of $\mathcal{S}$ and, as a consequence, we obtain the analog of Langlands' disjointness Theorem for real reductive groups: two standard tempered induced representations are either disjoint, i.e. without simple subquotient in common, or equivalent.

Then we discuss the characterisation of the Fourier transform, and of the set of minimal central idempotents of the reduced $C^{*}$-algebra $\mathcal{C}_{r}^{*}(\mathcal{H})$ of $\mathcal{H}$.

Finally we observe that that the dense subalgebra $\mathcal{S} \subset \mathcal{C}_{r}^{*}$ is closed for holomorphic calculus.

Let us now comment on the proof of the Main Theorem. As it is familiar since Harish-Chandra's work on real reductive groups [7], 8], 
the determination of the image of $\mathcal{S}$ by $\mathcal{F}$ requires a theory of the constant term (see also [5] for the case of the hypergeometric Fourier transform) for coefficients of tempered representations of $\mathcal{H}$. This theory is fairly simple using the decomposition of these linear forms on $\mathcal{H}$ along weights of the action of the abelian subalgebra $\mathcal{A}$ of $\mathcal{H}$. This subalgebra admits as a basis, the family $\theta_{x}, x \in X$, which arises in the Bernstein presentation of $\mathcal{H}$.

There is a natural candidate $\hat{\mathcal{S}}$ for the image of $\mathcal{S}$ by $\mathcal{F}$. The inclusion $\mathcal{F}(\mathcal{S}) \subset \hat{\mathcal{S}}$ is easy to prove, using estimates of the coefficients of standard induced tempered representations.

The only thing that remains to be proved at this point, is that the inverse of the Fourier transform, the wave packet operator $\mathcal{J}$, maps $\hat{\mathcal{S}}$ to $\mathcal{S}$. For this a particular role is played by normalized smooth family of coefficients of standard tempered induced representations: these are smooth families divided by the $c$-function. Of particular importance is the fact that the constant terms of these families are finite sums of normalized smooth families of coefficients for Hecke subalgebras of smaller semisimple rank. This is a nontrivial fact which requires the explicit computation of the constant term of coefficients for generic standard tempered induced representations. If $\mathcal{I}$ is the maximal ideal of the center $\mathcal{Z}$ of $\mathcal{H}$ which annihilates such a representation, its coefficients can be viewed as linear forms on Lusztig's formal completion of $\mathcal{H}$ associated to $\mathcal{I}$. This allows to use Lusztig's first reduction Theorem 11 which decomposes this algebra. Some results on Weyl groups are then needed to achieve this computation of the constant term.

Once this property of normalized smooth family is obtained, it easy to form wave packets in the Schwartz space, by analogy with HarishChandra's work for real reductive groups [7. Simple lemmas on spectral projections of matrices and an induction argument, allowed by the theory of the constant term, lead to the desired result.

The paper is roughly structured as follows. First we discuss in Sections 2 and 3 the necessary preliminary material on the affine Hecke algebra and the Fourier transform on $L_{2}(\mathcal{H})$. We formulate the Main Theorem in Section 4, and we discuss some of its consequences. In Section 5 we compute the constant terms of coefficients of the standard induced representations and of normalized smooth families of such coefficients. In Section 6 we use this and the material in the Appendix on spectral projections in order to prove the Main Theorem. Finally, in the Appendix on the $c$-function we have collected some fundamental properties of the Macdonald $c$-functions on which many of our results ultimately rely. 


\section{The affine Hecke algebra and the Schwartz alge- bra}

This section serves as a reminder for the definition of the affine Hecke algebra and related analytic structures. We refer the reader to [14, 11] and 13 for further background material.

\subsection{The root datum and the affine Weyl group}

A reduced root datum is a 5-tuple $\mathcal{R}=\left(X, Y, R_{0}, R_{0}^{\vee}, F_{0}\right)$, where $X, Y$ are lattices with perfect pairing $\langle\cdot, \cdot\rangle, R_{0} \subset X$ is a reduced root system, $R_{0}^{\vee} \subset Y$ is the coroot system (which is in bijection with $R_{0}$ via the map $\alpha \rightarrow \alpha^{\vee}$ ), and $F_{0} \subset R_{0}$ is a basis of simple roots of $R_{0}$. The set $F_{0}$ determines a subset $R_{0,+} \subset R_{0}$ of positive roots.

The Weyl group $W_{0}=W\left(R_{0}\right) \subset \mathrm{GL}(X)$ of $R_{0}$ is the group generated by the reflections $s_{\alpha}$ in the roots $\alpha \in R_{0}$. The set $S_{0}:=\left\{s_{\alpha} \mid \alpha \in F_{0}\right\}$ is called the set of simple reflections of $W_{0}$. Then $\left(W_{0}, S_{0}\right)$ is a finite Coxeter group.

We define the affine Weyl group $W=W(\mathcal{R})$ associated to $\mathcal{R}$ as the semidirect product $W=W_{0} \ltimes X$. The lattice $X$ contains the root lattice $Q$, and the normal subgroup $W^{\text {aff }}:=W_{0} \ltimes Q \triangleleft W$ is a Coxeter group whose Dynkin diagram is given by the affine extension of (each component of) the Dynkin diagram of $R_{0}^{\vee}$. The affine root system of $W^{\text {aff }}$ is given by $R^{\text {aff }}=R_{0}^{\vee} \times \mathbb{Z} \subset Y \times \mathbb{Z}$. Note that $W$ acts on $R^{\text {aff }}$.

Let $R_{+}^{\text {aff }}$ be the set of positive affine roots defined by $R_{+}^{\text {aff }}=\left\{\left(\alpha^{\vee}, n\right) \mid\right.$ $n>0$, or $n=0$ and $\left.\alpha \in R_{0,+}\right\}$. Let $F^{\text {aff }}$ denote the corresponding set of affine simple roots. Observe that $F_{0}^{\vee} \subset F^{\text {aff }}$. If $S^{\text {aff }}$ denotes the associated set of affine simple reflections, then ( $\left.W^{\text {aff }}, S^{\text {aff }}\right)$ is an affine Coxeter group.

In this paper we adhere to the convention $\mathbb{N}=\{1,2,3, \cdots\}$ and $\mathbb{Z}_{+}=\{0,1,2, \ldots\}$. We define the length function $l: W \rightarrow \mathbb{Z}_{+}$on $W$ as usual, by means of the formula $l(w):=\left|R_{+}^{\text {aff }} \cap w^{-1}\left(R_{-}^{\text {aff }}\right)\right|$. Let $\Omega \subset W$ denote the set $\{w \in W \mid l(w)=0\}$. It is a subgroup of $W$, complementary to $W^{\text {aff }}$. Therefore $\Omega \simeq X / Q$ is a finitely generated Abelian subgroup of $W$.

Let $X^{+} \subset X$ denote the cone of dominant elements $X^{+}=\{x \in X \mid$ $\left.\forall \alpha \in R_{0,+}:\left\langle x, \alpha^{\vee}\right\rangle \geq 0\right\}$. Then $Z_{X}:=X^{+} \cap X^{-} \subset X$ is a sublattice which is central in $W$. In particular it follows that $Z_{X} \subset \Omega$. The quotient $\Omega_{f} \simeq \Omega / Z_{X}$ is a finite Abelian group which acts faithfully on $S^{\text {aff }}$ by means of diagram automorphisms.

We choose a basis $z_{i}$ of $Z_{X}$, and define a norm $\|\cdot\|$ on the rational vector space $\mathbb{Q} \otimes_{\mathbb{Z}} Z_{X}$ by $\left\|\sum l_{i} z_{i}\right\|:=\sum\left|l_{i}\right|$. We now define a norm $\mathcal{N}$ 
on $W$ by

$$
\mathcal{N}(w):=l(w)+\left\|w(0)^{0}\right\|
$$

where $w(0)^{0}$ denotes the projection of $w(0)$ onto $\mathbb{Q} \otimes_{\mathbb{Z}} Z_{X}$ along $\mathbb{Q} \otimes_{\mathbb{Z}} Q$. The norm $\mathcal{N}$ plays an important role in this paper. Observe that it satisfies

$$
\mathcal{N}\left(w w^{\prime}\right) \leq \mathcal{N}(w)+\mathcal{N}\left(w^{\prime}\right),
$$

and that $\mathcal{N}(w)=0$ iff $w$ is an element of $\Omega$ of finite order.

We call $\mathcal{R}$ semisimple if $Z_{X}=0$.

\subsection{Standard parabolic subsystems}

A root subsystem $R^{\prime} \subset R_{0}$ is called parabolic if $R^{\prime}=\mathbb{Q} R^{\prime} \cap R_{0}$. The Weyl group $W_{0}$ acts on the collection of parabolic root subsystems. Let $\mathcal{P}$ be the power set of $F_{0}$. With $P \in \mathcal{P}$ we associate a standard parabolic root subsystem $R_{P} \subset R_{0}$ by $R_{P}:=\mathbb{Z} P \cap R_{0}$. Every parabolic root subsystem is $W_{0}$-conjugate to a standard parabolic subsystem.

We denote by $W_{P}=W\left(R_{P}\right) \subset W_{0}$ the Coxeter subgroup of $W_{0}$ generated by the reflections in $P$. We denote by $W^{P}$ the set of shortest length representatives of the left cosets $W_{0} / W_{P}$ of $W_{P} \subset W_{0}$.

Given $P \in \mathcal{P}$ we define a sub root datum $\mathcal{R}^{P} \subset \mathcal{R}$ simply by $\mathcal{R}^{P}:=$ $\left(X, Y, R_{P}, R_{P}^{\vee}, P\right)$. We also define a "quotient root datum" $\mathcal{R}_{P}$ of $\mathcal{R}^{P}$ by $\mathcal{R}_{P}=\left(X_{P}, Y_{P}, R_{P}, R_{P}^{\vee}, P\right)$ where $X_{P}:=X /\left(X \cap\left(R_{P}^{\vee}\right)^{\perp}\right)$ and $Y_{P}=$ $Y \cap \mathbb{Q} R_{P}^{\vee}$. The root datum $\mathcal{R}_{P}$ is semisimple.

\subsection{Label functions and root labels}

A positive real label function is a length multiplicative function $q$ : $W \rightarrow \mathbb{R}_{+}$. This means that $q\left(w w^{\prime}\right)=q(w) q\left(w^{\prime}\right)$ whenever $l\left(w w^{\prime}\right)=$ $l(w)+l\left(w^{\prime}\right)$, and that $q(\omega)=1$ for all $\omega \in \Omega$.

Such a function $q$ is uniquely determined by its restriction to the set of affine simple reflections $S^{\text {aff }}$. By the braid relations and the action of $\Omega_{f}$ on $S^{\text {aff }}$ it follows easily that $q(s)=q\left(s^{\prime}\right)$ whenever $s, s^{\prime} \in S^{\text {aff }}$ are $W$-conjugate. Hence there exists a unique $W$-invariant function $a \rightarrow q_{a}$ on $R^{\text {aff }}$ such that $q_{a+1}=q\left(s_{a}\right)$ for all simple affine roots $a \in F^{\text {aff }}$.

We associate a possibly non-reduced root system $R_{\mathrm{nr}}$ with $\mathcal{R}$ by

$$
R_{\mathrm{nr}}:=R_{0} \cup\left\{2 \alpha \mid \alpha^{\vee} \in R_{0}^{\vee} \cap 2 Y\right\} .
$$

If $\alpha \in R_{0}$ then $2 \alpha \in R_{\mathrm{nr}}$ iff the affine roots $a=\alpha^{\vee}$ and $a=\alpha^{\vee}+1$ are not $W$-conjugate. Therefore we can also characterize the label function 
$q$ on $W$ by means of the following extension of the set of root labels $q_{\alpha} \vee$ to arbitrary $\alpha \in R_{\mathrm{nr}}$. If $\alpha \in R_{0}$ with $2 \alpha \in R_{\mathrm{nr}}$, then we define

$$
q_{\alpha \vee / 2}:=\frac{q_{\alpha^{\vee}+1}}{q_{\alpha \vee}}
$$

With these conventions we have for all $w \in W_{0}$

$$
q(w)=\prod_{\alpha \in R_{\mathrm{nr},+} \cap w^{-1} R_{\mathrm{nr},-}} q_{\alpha^{\vee}}
$$

We denote by $R_{1} \subset X$ the reduced root system

$$
R_{1}:=\left\{\alpha \in R_{\mathrm{nr}} \mid 2 \alpha \notin R_{\mathrm{nr}}\right\}
$$

2.3.1. Restriction to parabolic subsystems. Let $P \in \mathcal{P}$. Both the nonreduced root system associated with $\mathcal{R}^{P}$ and the non-reduced root system associated with $\mathcal{R}_{P}$ are equal to $R_{P, n r}:=\mathbb{Q} R_{P} \cap R_{\mathrm{nr}}$. We define a collection of root labels $q_{P, \alpha} \vee=q_{\alpha \vee}^{P}$ for $\alpha \in R_{P, \mathrm{nr}}$ by restricting the labels of $R_{\mathrm{nr}}$ to $R_{P, \mathrm{nr}} \subset R_{\mathrm{nr}}$. Then $q_{P}$ denotes the length-multiplicative function on $W\left(\mathcal{R}_{P}\right)$ associated with this label function on $R_{P, \mathrm{nr}}$, and $q^{P}$ denotes the associated length multiplicative function on $W\left(\mathcal{R}^{P}\right)$.

\subsection{The Iwahori-Hecke algebra}

Given a root datum $\mathcal{R}$ and a (positive real) label function $q$ on the associated affine Weyl group $W$, there exists a unique associative complex Hecke algebra $\mathcal{H}=\mathcal{H}(\mathcal{R}, q)$ with $\mathbb{C}$-basis $N_{w}$ indexed by $w \in$ $W$, satisfying the relations

(i) $N_{w w^{\prime}}=N_{w} N_{w^{\prime}}$ for all $w, w^{\prime} \in W$ such that $l\left(w w^{\prime}\right)=l(w)+$ $l\left(w^{\prime}\right)$.

(ii) $\left(N_{s}+q(s)^{-1 / 2}\right)\left(N_{s}-q(s)^{1 / 2}\right)=0$ for all $s \in S^{\text {aff }}$.

Notice that the algebra $\mathcal{H}$ is unital, with unit $1=N_{e}$. Notice also that it follows from the defining relations that $N_{w} \in \mathcal{H}$ is invertible, for all $w \in W$.

By convention we assume that the label function $q$ is of the form

$$
q(s)=\mathbf{q}^{f_{s}} .
$$

The parameters $f_{s} \in \mathbb{R}$ are fixed, and the base $\mathbf{q}$ satisfies $\mathbf{q}>1$.

2.4.1. Bernstein presentation. There is another, extremely important presentation of the algebra $\mathcal{H}$, due to Joseph Bernstein (unpublished). Since the length function is additive on the dominant cone $X^{+}$, the map $X^{+} \ni x \rightarrow N_{x}$ is a homomorphism of the commutative monoid $X^{+}$with values in $\mathcal{H}^{\times}$, the group of invertible elements of $\mathcal{H}$. Thus 
there exists a unique extension to a homomorphism $X \ni x \rightarrow \theta_{x} \in \mathcal{H}^{\times}$ of the lattice $X$ with values in $\mathcal{H}^{\times}$.

The Abelian subalgebra of $\mathcal{H}$ generated by $\theta_{x}, x \in X$, is denoted by $\mathcal{A}$. Let $\mathcal{H}_{0}=\mathcal{H}\left(W_{0}, q_{0}\right)$ be the finite type Hecke algebra associated with $W_{0}$ and the restriction $q_{0}$ of $q$ to $W_{0}$. Then the Bernstein presentation asserts that both the collections $\theta_{x} N_{w}$ and $N_{w} \theta_{x}\left(w \in W_{0}, x \in X\right)$ are bases of $\mathcal{H}$, subject only to the cross relation (for all $x \in X$ and $s=s_{\alpha}$ with $\alpha \in F_{0}$ ):

$$
\begin{aligned}
& \theta_{x} N_{s}-N_{s} \theta_{s(x)}= \\
& \left\{\begin{array}{cl}
\left(q_{\alpha^{\vee}}^{1 / 2}-q_{\alpha \vee}^{-1 / 2}\right) \frac{\theta_{x}-\theta_{s(x)}}{1-\theta_{-\alpha}} & \text { if } 2 \alpha \notin R_{\mathrm{nr}} . \\
\left(\left(q_{\alpha^{\vee} / 2}^{1 / 2} q_{\alpha^{\vee}}^{1 / 2}-q_{\alpha^{\vee} / 2}^{-1 / 2} q_{\alpha \vee}^{-1 / 2}\right)+\left(q_{\alpha^{\vee}}^{1 / 2}-q_{\alpha^{\vee}}^{-1 / 2}\right) \theta_{-\alpha}\right) \frac{\theta_{x}-\theta_{s(x)}}{1-\theta_{-2 \alpha}} & \text { if } 2 \alpha \in R_{\mathrm{nr}} .
\end{array}\right.
\end{aligned}
$$

2.4.2. The center $\mathcal{Z}$ of $\mathcal{H}$. A rather immediate consequence of the Bernstein presentation of $\mathcal{H}$ is the description of the center of $\mathcal{H}$ :

Theorem 2.1. The center $\mathcal{Z}$ of $\mathcal{H}$ is equal to $\mathcal{A}^{W_{0}}$. In particular, $\mathcal{H}$ is finitely generated over its center.

As an immediate consequence we see that irreducible representations of $\mathcal{H}$ are finite dimensional by application of (Dixmier's version of) Schur's lemma.

We denote by $T$ the complex torus $T=\operatorname{Hom}\left(X, \mathbb{C}^{\times}\right)$of complex characters of the lattice $X$. The space $\operatorname{Spec}(\mathcal{Z})$ of complex homomorphisms of $\mathcal{Z}$ is thus canonically isomorphic to the (geometric) quotient $W_{0} \backslash T$.

Thus, to an irreducible representation $(V, \pi)$ of $\mathcal{H}$ we attach an orbit $W_{0} t \in W_{0} \backslash T$, called the central character of $\pi$.

2.4.3. Parabolic subalgebras and their semisimple quotients. We consider another important consequence of the Bernstein presentation of $\mathcal{H}$ :

Proposition 2.2. $\quad$ (i) The Hecke algebra $\mathcal{H}^{P}:=\mathcal{H}\left(\mathcal{R}^{P}, q^{P}\right)$ is isomorphic to the subalgebra of $\mathcal{H}$ generated by $\mathcal{A}$ and the finite type Hecke subalgebra $\mathcal{H}\left(W_{P}\right):=\mathcal{H}\left(W_{P},\left.q\right|_{W_{P}}\right)$.

(ii) We can view $\mathcal{H}_{P}:=\mathcal{H}\left(\mathcal{R}_{P}, q_{P}\right)$ as a quotient of $\mathcal{H}^{P}$ via the surjective homomorphism $\phi_{1}: \mathcal{H}^{P} \rightarrow \mathcal{H}_{P}$ characterized by (1) $\phi_{1}$ is the identity on the finite type subalgebra $\mathcal{H}\left(W_{P}\right)$ and (2) $\phi_{1}\left(\theta_{x}\right):=\theta_{\bar{x}}$, where $\bar{x} \in X_{P}$ is the canonical image of $x$ in $X_{P}=X /\left(X \cap\left(R_{P}^{\vee}\right)^{\perp}\right)$.

Let $T^{P}$ denote the character torus of the lattice $X /\left(X \cap \mathbb{Q} R_{P}\right)$. Then $T^{P} \subset T$ is a subtorus which is fixed for all the elements $w \in W_{P}$ and 
which is inside the simultaneous kernel of the $\alpha \in R_{P}$. The next result again follows simply from the Bernstein presentation:

Proposition 2.3. There exists a family of automorphisms $\psi_{t}\left(t \in T^{P}\right)$ of $\mathcal{H}^{P}$, defined by $\psi_{t}\left(\theta_{x} N_{w}\right)=x(t) \theta_{x} N_{w}$.

We use the above family of automorphisms to twist the projection $\phi_{1}: \mathcal{H}^{P} \rightarrow \mathcal{H}_{P}$. Given $t \in T^{P}$, we define the epimorphism $\phi_{t}: \mathcal{H}^{P} \rightarrow$ $\mathcal{H}_{P}$ by $\phi_{t}:=\phi_{1} \circ \psi_{t}$.

\subsection{Intertwining elements}

When $s=s_{\alpha} \in S_{0}$ (with $\alpha \in F_{1}$ ), we define an intertwining element $\iota_{s} \in \mathcal{H}$ as follows:

$$
\begin{aligned}
\iota_{s} & =q_{\alpha^{\vee}} q_{2 \alpha^{\vee}}\left(1-\theta_{-\alpha}\right) N_{s}+\left(\left(1-q_{\alpha^{\vee}} q_{2 \alpha^{\vee}}\right)+q_{\alpha^{\vee}}^{1 / 2}\left(1-q_{2 \alpha^{\vee}}\right) \theta_{-\alpha / 2}\right) \\
& =q_{\alpha^{\vee}} q_{2 \alpha^{\vee}} N_{s}\left(1-\theta_{\alpha}\right)+\left(\left(q_{\alpha^{\vee}} q_{2 \alpha^{\vee}}-1\right) \theta_{\alpha}+q_{\alpha^{\vee}}^{1 / 2}\left(q_{2 \alpha^{\vee}}-1\right) \theta_{\alpha / 2}\right)
\end{aligned}
$$

(If $\alpha / 2 \notin X$ then we put $q_{2 \alpha^{\vee}}=1$; see Remark 8.1.) We recall from [13], Theorem 2.8 that these elements satisfy the braid relations, and they satisfy (for all $x \in X$ )

$$
\iota_{s} \theta_{x}=\theta_{s(x)} \iota_{s}
$$

Let $\mathcal{Q}$ denote the quotient field of the centre $\mathcal{Z}$ of $\mathcal{H}$, and let ${ }_{\mathcal{Q}} \mathcal{H}$ denote the $\mathcal{Q}$-algebra ${ }_{\mathcal{Q}} \mathcal{H}=\mathcal{Q} \otimes_{\mathcal{Z}} \mathcal{H}$. Inside ${ }_{\mathcal{Q}} \mathcal{H}$ we normalize the elements $\iota_{s}$ as follows.

We first introduce

$$
n_{\alpha}:=\left(q_{\alpha^{\vee}}^{1 / 2}+\theta_{-\alpha / 2}\right)\left(q_{\alpha \vee}^{1 / 2} q_{2 \alpha^{\vee}}-\theta_{-\alpha / 2}\right) \in \mathcal{A} .
$$

Then the normalized intertwiners $\iota_{s}^{0}\left(s \in S_{0}\right)$ are defined by (with $\left.s=s_{\alpha}, \alpha \in R_{1}\right)$ :

$$
\iota_{s}^{0}:=n_{\alpha}^{-1} \iota_{s} \in \mathcal{Q}^{\mathcal{H}}
$$

It is known that the normalized elements $\iota_{s}^{0}$ satisfy $\left(\iota_{s}^{0}\right)^{2}=1$. In particular, $\iota_{s}^{0} \in \mathcal{Q}_{\mathcal{H}}{ }^{\times}$, the group of invertible elements of $\mathcal{Q}_{\mathcal{H}}$. In fact we have:

Lemma 2.4. (14, Lemma 4.1) The map $S_{0} \ni s \rightarrow \iota_{s}^{0} \in{ }_{\mathcal{Q}} \mathcal{H}^{\times}$extends (uniquely) to a homomorphism $W_{0} \ni w \rightarrow \iota_{w}^{0} \in \mathcal{Q} \mathcal{H}^{\times}$. Moreover, for all $f \in{ }_{\mathcal{Q}} \mathcal{A}$ we have that $\iota_{w}^{0} f \iota_{w^{-1}}^{0}=f^{w}$. 


\subsection{Hilbert algebra structure on $\mathcal{H}$}

The anti-linear map $h \rightarrow h^{*}$ defined by $\left(\sum_{w} c_{w} N_{w}\right)^{*}=\sum_{w} \bar{c}_{w^{-1}} N_{w}$ is an anti-involution of $\mathcal{H}$. Thus it gives $\mathcal{H}$ the structure of an involutive algebra.

In the context of involutive algebras we also dispose of Schur's lemma for topologically irreducible representations (cf. [6]). Thus the topologically irreducible representations of the involutive algebra $(\mathcal{H}, *)$ are finite dimensional by Theorem 2.1.

The linear functional $\tau: \mathcal{H} \rightarrow \mathbb{C}$ given by $\tau\left(\sum_{w} c_{w} N_{w}\right)=c_{e}$ is a positive trace for the involutive algebra $(\mathcal{H}, *)$. The basis $N_{w}$ of $\mathcal{H}$ is orthonormal with respect to the pre-Hilbert structure $(x, y):=\tau\left(x^{*} y\right)$ on $\mathcal{H}$. We denote the Hilbert completion of $\mathcal{H}$ with respect to $(\cdot, \cdot)$ by $L_{2}(\mathcal{H})$. This is a separable Hilbert space with Hilbert basis $N_{w}$ $(w \in W)$.

Let $x \in \mathcal{H}$. The operators $\lambda(x): \mathcal{H} \rightarrow \mathcal{H}$ (given by $\lambda(x)(y)=x y$ ) and $\rho(x): \mathcal{H} \rightarrow \mathcal{H}$ (given by $\rho(x)(y):=x y$ ) extend to $B\left(L_{2}(\mathcal{H})\right.$ ), the algebra of bounded operators on $L_{2}(\mathcal{H})$. This gives $\mathcal{H}$ the structure of a Hilbert algebra (cf. [6]).

The operator norm completion of $\lambda(\mathcal{H}) \subset B\left(L_{2}(\mathcal{H})\right)$ is a $C^{*}$-algebra which we call the reduced $C^{*}$-algebra $C_{r}^{*}(\mathcal{H})$ of $\mathcal{H}$. The natural action of $C_{r}^{*}(\mathcal{H})$ on $L_{2}(\mathcal{H})$ via $\lambda$ (resp. $\rho$ ) is called the left regular (resp. right regular) representation of $C_{r}^{*}(\mathcal{H})$. Since it has only finite dimensional irreducible representations by the above remark, $C_{r}^{*}(\mathcal{H})$ is of type I.

The norm $\|x\|_{o}$ of $x \in C_{r}^{*}(\mathcal{H})$ is by definition equal to the norm of $\lambda(x) \in B\left(L_{2}(\mathcal{H})\right)$. Observe that the map $x \rightarrow \lambda(x) N_{e}$ defines an embedding $C_{r}^{*}(\mathcal{H}) \subset L_{2}(\mathcal{H})$.

\subsection{Discrete series representations}

Definition 2.5. We call an irreducible representation $\left(V_{\delta}, \delta\right)$ of $(\mathcal{H}, *)$ a discrete series representation if $\left(V_{\delta}, \delta\right)$ is equivalent to a subrepresentation of $\left(L_{2}(\mathcal{H}), \lambda\right)$. We denote by $\Delta=\Delta_{\mathcal{R}, q}$ a complete set of representatives of the equivalence classes of the irreducible discrete series representations of $(\mathcal{H}, *)$. When $r \in T$ is given, $\Delta_{W_{0} r} \subset \Delta$ denotes the subset of $\Delta$ consisting of irreducible discrete series representations with central character $W_{0} r(r \in T)$.

Corollary 2.6. (of Theorem [2.1) $\Delta_{W_{0} r}$ is a finite set.

There is an important characterization of the discrete series representations due to Casselman. This characterization has consequences for the growth behaviour of matrix coefficients of discrete series representations. Recall that $T$ denotes the complex algebraic torus of characters 
of the lattice $X$. It has polar decomposition $T=T_{r s} T_{u}$ where $T_{r s}$ is the real split form of $T$, and $T_{u}$ the compact form. If $t \in T$ we denote by $|t| \in T_{r s}$ its real split part.

Theorem 2.7. (Casselman's criterion for discrete series representations, cf. 14, Lemma 2.22). Let $\left(V_{\delta}, \delta\right)$ be an irreducible representation of $\mathcal{H}$. The following are equivalent:

(i) $\left(V_{\delta}, \delta\right)$ is a discrete series representation.

(ii) All matrix coefficients of $\delta$ belong to $\mathfrak{H}$.

(iii) The character $\chi$ of $\delta$ belongs to $\mathfrak{H}$.

(iv) The weights $t \in T$ of the generalized $\mathcal{A}$-weight spaces of $V_{\delta}$ satisfy: $|x(t)|<1$, for all $0 \neq x \in X^{+}$.

(v) $Z_{X}=\{0\}$, and there exists an $\epsilon>0$ such that for all matrix coefficients $m$ of $\delta$, there exists a $C>0$ such that the inequality $\left|m\left(N_{w}\right)\right|<C \mathbf{q}^{-\epsilon l(x)}$ holds.

We have the following characterization of the set of central characters of irreducible discrete series representations. For the notion of "residual points" of $T$ we refer the reader to Definition 8.3 .

Theorem 2.8. (cf. 14, Lemma 3.31 and Corollary 7.12) The set $\Delta_{W_{0} r}$ is nonempty iff $r \in T$ is a residual point. In particular, $\Delta$ is finite, and empty unless $Z_{X}=0$.

\subsection{The Schwartz algebra; tempered representations}

We define norms $p_{n}\left(n \in \mathbb{Z}_{+}=\{0,1,2, \ldots\}\right)$ on $\mathcal{H}$ by

$$
p_{n}(h)=\max _{w \in W}\left|\left(N_{w}, h\right)\right|(1+\mathcal{N}(w))^{n},
$$

and we define the Schwartz completion $\mathcal{S}$ of $\mathcal{H}$ by

$$
\mathcal{S}:=\left\{x=\sum_{w} x_{w} N_{w} \in \mathcal{H}^{*} \mid p_{n}(x)<\infty \forall n \in \mathbb{Z}_{+}\right\}
$$

In (14, Theorem 6.5) it was shown that the multiplication operation of $\mathcal{H}$ is continuous with respect to the family $p_{n}$ of norms. The completion $\mathcal{S}$ is a (nuclear, unital) Fréchet algebra (cf. 14, Definition 6.6).

As an application of ([14, Theorem 6.1) it is easy to see that there exist constants $D \in \mathbb{Z}_{+}$and $C>0$ such that $\|h\|_{o} \leq C p_{D}(h)$ for all $h \in \mathcal{H}$. Thus

$$
\mathcal{S} \subset C_{r}^{*}(\mathcal{H}) \subset L_{2}(\mathcal{H})
$$

The Main Theorem 4.3 can be viewed as a structure theorem for this Fréchet algebra via the Fourier transformation. 
Definition 2.9. The topological dual $\mathcal{S}^{\prime}$ is called the space of tempered functionals. A continuous representation of $\mathcal{S}$ is called a tempered representation. By abuse of terminology, we call a finite dimensional representation of $\mathcal{H}$ tempered if it extends continuously to $\mathcal{S}$.

In particular, a finite dimensional representation $(V, \pi)$ of $\mathcal{H}$ is tempered if and only if the matrix coefficient $h \rightarrow \phi(\pi(h) v)$ extends continuously to $\mathcal{S}$ for all $\phi \in V^{*}$ and $v \in V$.

We will now discuss Casselman's criteria for temperedness of finite functionals and finite dimensional representations if $\mathcal{H}$.

\subsection{Casselman's criteria for temperedness}

2.9.1. Algebraic dual of $\mathcal{H}$. We identify the algebraic dual $\mathcal{H}^{*}$ of $\mathcal{H}$ with formal linear combinations $f=\sum_{w \in W} d_{w} N_{w}$ via the sesquilinear pairing $(\cdot, \cdot)$ defined by $(x, y)=\tau\left(x^{*} y\right)$. Thus $f(x)=\left(f^{*}, x\right)$ and $d_{w}=f\left(N_{w^{-1}}\right)$. For $x, y \in \mathcal{H}$ and $f \in \mathcal{H}$ we define $R_{x}(f)(y)=f(y x)$ and $L_{x}(f)(y):=f(x y)$ (a right representation of $\mathcal{H}$ ). Note that in terms of multiplication of formal series we have: $R_{x}(f)=x . f$ and $L_{x}(f)=f . x(\mathrm{sic})$.

2.9.2. Finite functionals. Let $\mathbb{A} \subset \mathcal{H}^{*}$ denote the linear space of finite linear functionals on $\mathcal{H}$ :

Definition 2.10. The space $\mathbb{A}$ consists of all the elements $f \in \mathcal{H}^{*}$ such that the space $\mathcal{H}$.f. $\mathcal{H}$ is finite dimensional.

Since $\mathcal{H}$ is finite over its center $\mathcal{Z}, f$ is finite if and only if $\operatorname{dim}(f . \mathcal{Z})<$ $\infty$. Let $\mathcal{A}$ denote the abelian subalgebra of $\mathcal{H}$ spanned by the elements $\theta_{x}$ with $x \in X$. Since $\mathcal{Z} \subset \mathcal{A}$ we see that $f \in \mathbb{A}$ if and only if $\operatorname{dim}(\mathcal{A} . f)<\infty$ if and only if $\operatorname{dim}(f . \mathcal{A})<\infty$.

\subsection{Exponents of finite functionals}

Definition 2.11. We say that $t \in T$ is an exponent of $f \in \mathbb{A}$ if the $X$-module on the finite dimensional space $V=f . \mathcal{H}$ (the space of left translates of $f$ ) defined via $\left.x \rightarrow L_{\theta_{x}}\right|_{V}$ contains a (generalized) weight space with weight $t$.

Proposition 2.12. Let $f \in \mathbb{A}$ and let $\epsilon$ denote the set of exponents of $f$. There exist unique functions $E_{t}^{f}(t \in \epsilon)$ on $\mathcal{H} \times X$, polynomial in $X$, such that

$$
f\left(\theta_{x} h\right)=\sum_{t \in \epsilon} E_{t}^{f}(h, x) t(x)
$$


Proof. Uniqueness: Suppose that we have a finite set $\epsilon$ of exponents and for each $t \in \epsilon$ a polynomial function $x \rightarrow E_{t}(x)$ of $X$ such that

$$
\sum_{t \in \epsilon} E_{t}(x) t(x) \equiv 0
$$

Suppose that there exists a $t \in \epsilon$ such that $x \rightarrow E_{t}(x)$ has positive degree. We apply the difference operator $\Delta_{t, y}(t \in \epsilon, y \in X)$ defined by

$$
\Delta_{t, y}(f)(x):=t(y)^{-1} f(x+y)-f(x) .
$$

It is easy to see that for a suitable choice of $y$ this operator lowers the degree of the coefficient of $t$ by 1 , and leaves the degrees of the other coefficients invariant. Hence, if we assume that not all of the coefficients $E_{t}$ are zero, we obtain a nontrivial complex linear relation of characters of $X$, after applying a suitable sequence of operators $\Delta_{s, z}$. This is a contradiction.

Existence: We fix $h \in \mathcal{H}$ and we decompose $f$ according to generalized $L_{X}$-eigenspaces in $V$. We may replace $f$ by one of its constituents, and thus assume that $\epsilon=\{t\}$. We may replace the action of $X$ by the action $L_{x}^{\prime}=t(x)^{-1} L_{x}$. Therefore it is enough to consider the case $t=1$. Let $N$ denote the dimension of $V$. By Engel's theorem applied to the commuting unipotent elements $L_{\theta_{x}}$ acting in $V$, we see that any product of $N$ or more difference operators of the form $\Delta_{y}=L_{\theta_{y}}-1$ is equal to zero in $V$. By induction on $N$ this implies that for any $h$, the function $x \rightarrow f\left(\theta_{x} h\right)$ is a polynomial in $x$ of degree at most $N-1$.

Corollary 2.13. We have $E_{t}^{f}\left(\theta_{x} h, y\right)=t(x) E_{t}^{f}(h, x+y)$. In particular, the degree of the polynomial $E_{t}^{f}(h, x)$ is uniformly bounded as a function of $h$.

Corollary 2.14. Put $f_{t}(h)=E_{t}^{f}(h, 0)$. Then $f_{t}$ is the component of $f$ corresponding to the generalized $L_{X}$-eigenspace with eigenvalue $t$ in $V$. Observe that $f_{t}\left(\theta_{x} h\right)=t(x) E_{t}^{f}(h, x)$, and that $f_{t} \in f \cdot \mathcal{A}=L_{X}(f) \subset$ $V \subset \mathbb{A}$.

\subsection{The space $\mathbb{A}^{t e m p}$ of tempered finite functionals}

If $f \in \mathbb{A}$, we can express the condition $f \in \mathcal{S}^{\prime}$ (temperedness) or $f \in L_{2}(\mathcal{H})$ (square integrability) in terms of a system of inequalities on the set of exponents $\epsilon$ of $f$. This is the content of the Casselman conditions for temperedness ([14], Lemma 2.20). We will formulate these results below, adapted to suit the applications we have in mind (Section 5.1). 
We introduce a partial ordering $\leq_{F_{0}}$ on $T_{r s}$ by

$$
t_{1} \leq_{F_{0}} t_{2} \Longleftrightarrow x\left(t_{1}\right) \leq x\left(t_{2}\right) \text { for all } x \in X^{+}
$$

(this is in fact the special case $P=F_{0}$ of the ordering $\leq_{P}$ defined in Definition 5.1).

Let $(V, \pi)$ be finite dimensional representation of $\mathcal{H}$. It follows easily from Definition 2.11 that the union of the sets of exponents of the matrix coefficients $h \rightarrow \phi(\pi(h) v)$ of $\pi$ coincides with the set of weights $t$ of the generalized $\mathcal{A}$-weight spaces of $V$. Using ([14], Lemma 2.20) we get:

Corollary 2.15. (14, Lemma 2.20) Let $(V, \pi)$ be a finite dimensional representation of $\mathcal{H}$. The following statements are equivalent:

(i) $(V, \pi)$ is tempered.

(ii) The weights $t$ of the generalized $\mathcal{A}$-weight spaces of $V$ satisfy $|t| \leq_{F_{0}} 1$.

(iii) The exponents $t$ of the matrix coefficients $h \rightarrow \phi(\pi(h) v)$ of $\pi$ satisfy $|t| \leq_{F_{0}} 1$.

Let $f \in \mathbb{A}$. The space of matrix coefficients of the finite dimensional representation $\left(V_{f}:=R_{\mathcal{H}}(f), R\right)$ is the space $\mathcal{H} \cdot f \cdot \mathcal{H}$. Hence the union of the sets of exponents of the matrix coefficients of $V_{f}$ is equal to the set of exponents of $f$. Hence we obtain:

Corollary 2.16. (Casselman's condition) We have $f \in \mathbb{A}^{\text {temp }}:=\mathbb{A} \cap \mathcal{S}^{\prime}$ if and only if the real part $|t|$ of every exponent $t$ of $f$ satisfies $|t| \leq_{F_{0}} 1$.

Definition 2.17. We put $\mathbb{A}^{\text {cusp }}$ for the subspace of $\mathbb{A}^{\text {temp }}$ consisting of those $f$ such that all exponents $t$ of $f$ satisfy $|t|=\prod_{\alpha \in F_{0}}\left(d_{a} \otimes \alpha^{\vee}\right)$ with $0<d_{\alpha}<1$.

Then Theorem 2.7 implies similarly that:

Corollary 2.18. $\mathbb{A}_{2}:=\mathbb{A} \cap L_{2}(\mathcal{H}) \neq 0$ only if $Z_{X}=0$, and in this case, $\mathbb{A}_{2}=\mathbb{A}^{\text {cusp }}$.

\subsection{Formal completion of $\mathcal{H}$ and Lusztig's structure theorem}

Let $t \in T$, and let $\mathcal{I}_{t}$ denote the maximal ideal of $\mathcal{Z}$ associated with the orbit $W_{0} t$. We denote by $\overline{\mathcal{Z}}_{W_{0} t}$ the $\mathcal{I}_{t}$-adic completion of $\mathcal{Z}$. In 11 Lusztig considered the structure of the completion

$$
\overline{\mathcal{H}}_{t}:=\overline{\mathcal{Z}}_{W_{0} t} \otimes_{\mathcal{Z}} \mathcal{H} .
$$

We will use Lusztig's results on the structure of this formal completion (in a slightly adapted version) for so called $R_{P}$-generic points $t \in T$. 


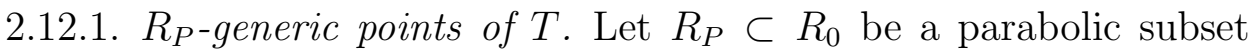
of roots, i.e. $R_{P}=\mathbb{R} R_{P} \cap R_{0}$. Let us recall the notion of an $R_{P^{-}}$ generic point $t \in T$ (cf. [14, Definition 4.12). With $t \in T$ we associate $R_{P(t)} \subset R_{0}$, the smallest parabolic subset containing all roots $\alpha \in R_{0}$ for which one of the following statements holds (where $c_{\alpha}$ denotes the Macdonald $c$-function, cf. equation (8.2)):

(i) $c_{\alpha} \notin \mathcal{O}_{t}^{\times}$(the invertible holomorphic germs at $t$ )

(ii) $\alpha(t)=1$

(iii) $\alpha(t)=-1$ and $\alpha \notin 2 X$.

We say that $t_{1}, t_{2} \in T$ are equivalent if there exists a $w \in W_{P\left(t_{1}\right)}:=$ $W\left(R_{P\left(t_{1}\right)}\right)$ such that $t_{2}=w\left(t_{1}\right)$. Notice that in this case $R_{P\left(t_{1}\right)}=R_{P\left(t_{2}\right)}$, so that this is indeed an equivalence relation. The equivalence class of $t \in T$ is equal to the orbit $\varpi=W_{P(t)} t \subset W_{0} t$.

We define $P(t)$ as the basis of simple roots of $R_{P(t)}$ inside $R_{0,+}$, and we sometimes use the notation $P(\varpi)$ instead of $P(t)$.

Definition 2.19. We call $t \in T$ an $R_{P}$-generic point if $w t \in \varpi$ (with $\left.w \in W_{0}\right)$ implies that $w \in W_{P}$.

Remark 2.20. Notice that if $t \in T$ is $R_{P}$ generic then $R_{P(t)} \subset R_{P}$ (but not conversely). In particular, the set of $R_{P}$ nongeneric points is contained in a finite union of cosets of the finite collection of codimension 1 complex subtori $H$ of $T$ such that $\alpha(H)=1$ for some $\alpha \in R_{0} \backslash R_{P}$.

2.12.2. Lusztig's first reduction Theorem. Lusztig 11 associates idempotents $e_{w \varpi} \in \overline{\mathcal{H}}_{t}$ with the equivalence classes $w \varpi \in W_{0} t$. By Lusztig's first reduction Theorem (cf. [11]) we know that if $u, v \in W^{P}$, then $\iota_{u}^{0} e_{\varpi} \iota_{v^{-1}}^{0}$ is a well defined element of $\overline{\mathcal{H}}_{t}$, and that we have the decomposition (compare with [14, equation (4.46))

$$
\overline{\mathcal{H}}_{t}=\bigoplus_{u, v \in W^{P}} \iota_{u}^{0} e_{\varpi} \overline{\mathcal{H}}_{t}^{P} \iota_{v^{-1}}^{0} .
$$

Moreover, the subspace $\iota_{u}^{0} e_{\varpi} \overline{\mathcal{H}}_{t}^{P} \iota_{v^{-1}}^{0}$ is equal to $e_{u \varpi} \overline{\mathcal{H}}_{t} e_{v \varpi}$. When $u=$ $v$ then this is a subalgebra of $\mathcal{H}_{t}$, and when $u=v=e$ then this subalgebra reduces to $e_{\varpi} \overline{\mathcal{H}}_{t}^{P}$, which is isomorphic to $\overline{\mathcal{H}}_{t}^{P}$ via $x \rightarrow e_{\varpi} x$. Finally, for $x \in \overline{\mathcal{H}}_{t}^{P}$ we have the formula $\iota_{u}^{0}\left(e_{\varpi} x\right) \iota_{u^{-1}}^{0}=e_{u \varpi} \psi_{u}(x)$.

We will use this in the situation that $t \in T$ is is of the form $t=r_{P} t^{P}$ with $W_{P} r_{P} \subset T_{P}$ the central character of a discrete series representation $\left(V_{\delta}, \delta\right)$, and $t^{P} \in T^{P}$ (this is the case if $W_{0} t \subset T$ is the central character of a representation which is induced from $\left(V_{\delta}, \delta\right)$. Recall that in this situation $r_{P} \in T_{P}$ is an $\left(R_{P}, q_{P}\right)$-residual point (Theorem 2.8). Therefore, $R_{P(t)} \supset R_{P}$ ([14], Proposition 7.3), and $R_{P(t)}=R_{P}$ for an 
open dense subset of $T^{P}$ (the complement of a subvariety of codimension 1 in $T^{P}$ ). Thus $t=r_{P} t^{P}$ is $R_{P}$-generic iff $R_{P(t)}=R_{P}$, and then the equivalence class of $t$ is equal to $\varpi=W_{P} t$.

2.12.3. Application. We will use the above result (2.18) when analyzing a finite functional $f \in \mathbb{A}$ or a representation $\pi$ of $\mathcal{H}$ which contains a power $\mathcal{I}_{t}^{n}$ of $\mathcal{I}_{t}$ in its kernel.

We can thus view $f$ (or $\pi$ ) as a linear function on the quotient $\mathcal{H} / \mathcal{I}_{t}^{n} \mathcal{H}$. Since this quotient is finite dimensional (by Theorem 2.1), we have

$$
\mathcal{H} / \mathcal{I}_{t}^{n} \mathcal{H}=\overline{\mathcal{H}}_{t} / \mathcal{I}_{t}^{n} \overline{\mathcal{H}}_{t}
$$

In this way we can view $f$ (resp. $\pi$ ) as a functional (resp. representation) of the completion $\overline{\mathcal{H}}_{t}$. For example, this applies when $W_{0} t$ is the central character of an irreducible representation $\pi$. We can view $\pi$ as a representation of the quotient $\mathcal{H}^{t}:=\mathcal{H} / \mathcal{I}_{t} \mathcal{H}$ (the case $n=1$ of $(2.19)$ ), and the matrix coefficients of $\pi$ can be viewed as functionals on $\mathcal{H}^{t}$.

\section{Fourier Transform}

In this section we briefly review the Fourier transform on $L_{2}(\mathcal{H})$ as formulated in 14. The spectral data are organized in terms of the induction functor on the groupoid of unitary standard induction data $\mathcal{W}_{\Xi_{u}}$. Finally we formulate the Main Theorem 4.3 and discuss its applications.

\subsection{Induction from standard parabolic subquotient algebras}

Let $P \subset F_{0}$ and let $W_{P} \subset W_{0}$ be the standard parabolic subgroup of $W_{0}$ generated by the simple reflections $s_{\alpha}$ with $\alpha \in P$. Let $\mathcal{H}^{P} \subset \mathcal{H}$ be the subalgebra $\mathcal{H}^{P}:=\mathcal{H}\left(W_{P}\right) \cdot \mathcal{A} \subset \mathcal{H}$, and let $\mathcal{H}_{P}$ denote the quotient of $\mathcal{H}^{P}$ by the (two sided) ideal generated by the central elements $\theta_{x}-1$ where $x \in X$ is such that $\left\langle x, \alpha^{\vee}\right\rangle=0$ for all $\alpha \in P$. Then $\mathcal{H}_{P}$ is again an affine Hecke algebra, with root datum $\mathcal{R}_{P}=\left(R_{P}, X_{P}, R_{P}^{\vee}, Y_{P}, P\right)$, where $X_{P}=X / P^{\vee}, \perp$ and $Y_{P}=Y \cap \mathbb{R} P^{\vee}$, and root labels $q_{P}$ that are obtained by restriction from $R_{\mathrm{nr}}$ to $R_{P, \mathrm{nr}}$.

There exists a parameter family of homomorphisms $\phi_{t^{P}}: \mathcal{H}^{P} \rightarrow \mathcal{H}_{P}$ with $t^{P} \in T^{P} \subset T$, the subtorus with character lattice $X^{P}=X /(X \cap$ $\mathbb{R} P)$, defined by $\phi_{t^{P}}\left(\theta_{x} T_{w}\right)=x\left(t^{P}\right) \theta_{\bar{x}} T_{w}$, where $\bar{x} \in X_{P}$ denotes the canonical image of $x$ in $X_{P}$. The kernel of $\phi_{t^{P}}$ is the two-sided ideal 
generated by elements of the form $x\left(t^{P}\right)^{-1} \theta_{x}-1$, with $x \in X$ such that $\left\langle x, \alpha^{\vee}\right\rangle=0$ for all $\alpha \in P$.

Let $\left(V_{\delta}, \delta\right)$ be a discrete series representation of the subquotient Hecke algebra $\mathcal{H}_{P}$. Let $W_{P} r_{P}$ be the central character of $\delta$. It is known that $r_{P}$ is a residual point of $T_{P}$ (cf. [14], Lemma 3.31), the subtorus of $T$ with character lattice $X_{P}$.

Now let $t^{P} \in T_{u}^{P}$, and let $\delta_{t^{P}}$ denote the lift to $\mathcal{H}^{P}$ of $\delta$ via $\phi_{t^{P}}$. Then the induced representation $\pi=\pi\left(\mathcal{R}_{P}, W_{P} r_{P}, \delta, t^{P}\right)$ from the representation $\delta_{t^{P}}$ of $\mathcal{H}^{P}$ to $\mathcal{H}$ is a unitary, tempered representation (cf. [14], Proposition 4.19 and Proposition 4.20).

3.1.1. Compact realization of $\pi\left(R_{P}, W_{P} r_{P}, \delta, t^{P}\right)$. Put $\mathcal{H}\left(W^{P}\right) \subset \mathcal{H}$ for the finite dimensional linear subspace of $\mathcal{H}$ spanned by the elements $N_{w}$ with $w \in W^{P}$. Then

$$
\mathcal{H} \simeq \mathcal{H}\left(W^{P}\right) \otimes \mathcal{H}^{P},
$$

where the isomorphism is realized by the product map. Therefore we have the isomorphism

$$
\mathcal{H} \otimes_{\mathcal{H}^{P}} V_{\delta} \simeq i\left(V_{\delta}\right):=\mathcal{H}\left(W^{P}\right) \otimes V_{\delta} .
$$

We will use this isomorphism to identify the representation space of $\pi\left(P, W_{P} r_{P}, \delta, t^{P}\right)$ with $i\left(V_{\delta}\right)$. This realization of the induced representation is called the compact realization, by analogy with induced representations for reductive groups.

According to 14, Proposition 4.19, the representation $\pi\left(P, W_{P} r_{P}, \delta, t^{P}\right)$ is unitary (i.e. a $*$-representation) with respect to the Hermitian inner product

$$
\left\langle h_{1} \otimes v_{1}, h_{2} \otimes v_{2}\right\rangle=\tau\left(h_{1}^{*} h_{2}\right)\left(v_{1}, v_{2}\right),
$$

where $\left(v_{1}, v_{2}\right)$ denotes the inner product on the representation space $V_{\delta}$ of the discrete series representation $\left(V_{\delta}, \delta\right)$.

More generally, for $t^{P} \in T^{P}$ the Hermitian form $\langle\cdot, \cdot\rangle$ on $i\left(V_{\delta}\right)$ defines a nondegenerate sesquilinear pairing of $\mathcal{H}$-modules as follows:

$$
\pi\left(P, W_{P} r_{P}, \delta, \bar{t}^{-1}\right) \times \pi\left(P, W_{P} r_{P}, \delta, t^{P}\right) \rightarrow \mathbb{C} .
$$

\subsection{Groupoid of standard induction data}

Let $\mathcal{P}$ denote the power set of $F_{0}$. Let $\Xi$ (respectively $\Xi_{u}$ ) denote the set of all triples $\xi=\left(P, \delta, t^{P}\right)$ with $P \in \mathcal{P}, \delta$ an irreducible discrete series representation of $\mathcal{H}_{P}$ (with underlying vector space $V_{\delta}$ ), and $t^{P} \in$ $T^{P}$ (respectively $t^{P} \in T_{u}^{P}$ ). We denote the central character of $\delta$ by $W_{P} r_{P}$ 
Let $\mathcal{W}$ denote the finite groupoid whose set of objects is $\mathcal{P}$ and such that the set of arrows from $P$ to $Q(P, Q \in \mathcal{P})$ consists of $K_{Q} \times W(P, Q)$, where $K_{Q}=T_{Q} \cap T^{Q}$ and $W(P, Q)=\left\{w \in W_{0} \mid w(P)=Q\right\}$. The composition of arrows is defined by $\left(k_{1}, w_{1}\right)\left(k_{2}, w_{2}\right)=\left(k_{1} w_{1}\left(k_{2}\right), w_{1} w_{2}\right)$. This groupoid acts on $\Xi$ as follows. An element $g=k \times n \in K_{Q} \times$ $W(P, Q)$ of $\mathcal{W}_{\Xi}$ defines an algebra isomorphism $\psi_{g}: \mathcal{H}_{P} \rightarrow \mathcal{H}_{Q}$ as follows. An element $n \in W(P, Q)$ defines an isomorphism from the root datum $\left(\mathcal{R}_{P}, q_{P}\right)$ to $\left(\mathcal{R}_{Q}, q_{Q}\right)$, which determines an algebra isomorphism $\psi_{n}$. On the other hand, if $k \in K_{Q}$ then $\psi_{k}: \mathcal{H}_{Q} \rightarrow \mathcal{H}_{Q}$ is the automorphism defined by $\psi_{k}\left(\theta_{x} N_{w}\right)=k(x) \theta_{x} N_{w}$. Then $\psi_{g}$ is defined by the composition of these isomorphisms. We obtain a bijection $\Psi_{g}: \Delta_{W_{P} r_{P}} \rightarrow \Delta_{k^{-1} W_{Q} n\left(r_{P}\right)}$ (where $\Delta_{W_{P} r_{P}}=\Delta_{P, W_{P} r_{P}}$ denotes a complete set of representatives for the equivalence classes of irreducible discrete series representations of $\mathcal{H}_{P}$ with central character $\left.W_{P} r_{P}\right)$ characterized by the requirement $\Psi_{g}(\delta) \simeq \delta \circ \psi_{g}^{-1}$. The action of $\mathcal{W}$ on $\Xi$ is defined by: $g\left(P, \delta, t^{P}\right)=\left(Q, \Psi_{g}(\delta), g\left(t^{P}\right)\right)$, with $g\left(t^{P}\right):=k n\left(t^{P}\right)$.

Definition 3.1. The fibred product $\mathcal{W}_{\Xi}=\mathcal{W} \times_{\mathcal{P}} \Xi$ is called the groupoid of standard induction data. The full compact subgroupoid $\mathcal{W}_{\Xi, u}=\mathcal{W} \times_{\mathcal{P}}$ $\Xi_{u}$ is called the groupoid of tempered standard induction data.

Definition 3.2. An element $\xi=\left(P, \delta, t^{P}\right) \in \Xi$ is called generic if

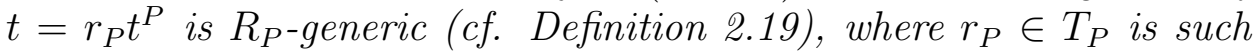
that $W_{P} r_{P}$ is the central character of $\delta$.

The groupoid $\mathcal{W}_{\Xi, u}$ was introduced in [14] (but was denoted by $\mathcal{W}_{\Xi}$ there) and plays an important role in the theory of the Fourier transform for $\mathcal{H}$. It is easy to see that $\mathcal{W}_{\Xi}$ is a smooth analytic, étale groupoid, whose set of objects is equal to $\Xi$. Thus $\mathcal{W}_{\Xi}$ is a union of complex algebraic tori, and therefore we can speak of polynomial and rational functions on $\Xi$ and on $\mathcal{W}_{\Xi}$. This also applies to the full compact subgroupoid $\mathcal{W}_{\Xi, u}$.

Theorem 4.38 of 14 states that there exists an induction functor $\pi: \mathcal{W}_{\Xi, u} \rightarrow \operatorname{PRep}_{\text {unit,temp }}(\mathcal{H})$, where the target groupoid is the category of finite dimensional, unitary, tempered representations of $\mathcal{H}$ in which the morphisms are given by unitary intertwining isomorphisms modulo the action of scalars. The image of $\xi=\left(P, \delta, t^{P}\right) \in \Xi_{u}$ is the representation $\pi(\xi):=\pi\left(P, W_{P} r_{P}, \delta, t^{P}\right)$ of $\mathcal{H}$, in its compact realization, as was defined in subsection 3.1.1.

The intertwining isomorphism $\pi(g, \xi): i\left(V_{\delta}\right) \rightarrow i\left(V_{\Psi_{g}(\delta)}\right)$ associated with $g=k \times n \in K_{Q} \times W(P, Q)$ is the operator $A\left(g, \mathcal{R}_{P}, W_{P} r_{P}, \delta, t^{P}\right)$ which was defined in [14] (equation (4.82)). In order to explain its construction we need to use Lusztig's theorem on the structure of the 
formal completion of $\mathcal{H}$ at the central character of $\pi(\xi)$ (cf. Subsection 2.12). The central character of $\pi(\xi)\left(\xi=\left(P, \delta, t^{P}\right)\right)$ is equal to $W_{0} t$ with $t=r_{P} t^{P}$, where $W_{P} r_{P}$ denotes the central character of $\delta$. Recall that we can then extend $\pi(\xi)$ to the formal completion $\overline{\mathcal{H}}_{t}$ of $\mathcal{H}$ with respect to the maximal ideal $\mathcal{I}_{t}$ of $\mathcal{Z}$ at $W_{0} t$ (cf. 2.12.3).

First we consider the case where $\xi$ is generic (Definition 3.2. Remark 2.20). For $w \in W^{P}, w \neq e$, the idempotent $e_{w w}$ (cf. equation 2.18) vanishes on $1 \otimes V_{\delta} \subset i\left(V_{\delta}\right)$, where the action is through $\pi(\xi)$ (extended to the completion). Therefore we have the natural isomorphisms of vector spaces:

$$
\begin{aligned}
i\left(V_{\delta}\right) & \simeq \mathcal{H} \otimes_{\mathcal{H}^{P}} V_{\delta} \\
& \simeq \overline{\mathcal{H}}_{t} \otimes_{e_{\varpi} \overline{\mathcal{H}}_{t}^{P}} V_{\delta} \\
& \simeq \bigoplus_{u \in W^{P}} \iota_{u}^{0} e_{\varpi} \otimes V_{\delta}
\end{aligned}
$$

where $e_{\varpi} \overline{\mathcal{H}}_{t}^{P} \simeq \overline{\mathcal{H}}_{t}^{P}$ acts on $V_{\delta}$ via $\delta_{t^{P}}$, extended to the formal completion at the central character $W_{P} t$. We will often suppress the subscript $e_{\varpi} \overline{\mathcal{H}}_{t}^{P}$ of $\otimes$.

Let us now define the unitary standard intertwining operators $\pi(g, \xi)$ in this case where $\xi$ is generic. First we choose a unitary isomorphism $\tilde{\delta}_{g}: V_{\delta} \rightarrow V_{\Psi_{g}(\delta)}$ intertwining the representations $\delta \circ \psi_{g}^{-1}$ and $\Psi_{g}(\delta)$. Then we define

$$
\begin{aligned}
\pi(g, \xi): i\left(V_{\delta}\right) & \rightarrow i\left(V_{\Psi_{g}(\delta)}\right) \\
h \otimes v & \rightarrow h \iota_{g^{-1}}^{0} e_{g \varpi} \otimes_{e_{g \varpi} \mathcal{H}_{g(t)}^{g(P)}} \tilde{\delta}_{g}(v),
\end{aligned}
$$

where we use the isomorphism of equation (3.5) to view the right hand side as an element of $i\left(V_{\Psi_{g}(\delta)}\right)$. It follows easily that $\pi(g, \xi)$ is an intertwining operator between $\pi(\xi)$ and $\pi(g \xi)$.

For general $\xi$ we need the following regularity results from [14. The matrix elements of $\pi(g, \xi)$ are meromorphic in $\xi$, with possible poles at the nongeneric $\xi$. However, it was shown in 14, Theorem 4.33, that for $R_{P^{-g e n e r i c ~}} t=r_{P} t^{P}, \pi(g, \xi)$ is unitary with respect to the Hilbert space structures of $i\left(V_{\delta}\right)$ and $i\left(V_{\Psi_{g}(\delta)}\right)$ (which are independent of $t^{P} \in T_{u}^{P}$, cf. equation (3.3) $)$. Together with a description of the locus of the possible singularities of $\pi(g, \xi)$ (as a rational function on $\Xi_{P, \delta}$, the set of induction data of the form $\left(P, \delta, t^{P}\right)$ with $\left.t^{P} \in T^{P}\right)$, this implies (according to a simple argument, cf. [2], Lemma 8) that $\pi(g, \xi)$ has only removable singularities in a tubular neighborhood of $\Xi_{P, \delta, u}$ (the subset of triples in $\Xi_{P, \delta}$ with $t^{P} \in T_{u}^{P}$ ). Thus $\pi(g, \xi)$ has a unique holomorphic extension to a tubular neighborhood of $\Xi_{P, \delta, u}$. 
This finally clarifies the definition of $\pi(g, \xi)$ for general $\xi \in \Xi_{P, \delta, u}$ (and in fact in a "tubular neighborhood" of this subset of $\Xi_{P, \delta}$ ).

We conclude with the following summary of the above

Theorem 3.3. The induction functor $\pi: \mathcal{W}_{\Xi, u} \rightarrow \operatorname{PRep}_{\text {unit,temp }}(\mathcal{H})$ is rational and smooth.

By this we simply mean that on each component $\Xi_{P, \delta, u}$ of $\Xi_{u}$, the representations $\pi(\xi)$ can be realized by smooth rational matrices as a function of $\xi \in \Xi_{P, \delta, u}$, and also the matrices of the $\pi(g, \xi)$ are both rational and smooth in $\xi \in \Xi_{P, \delta, u}$. We note that the matrices $\pi(\xi ; h):=$ $\pi(\xi)(h)$ (for $h \in \mathcal{H}$ fixed) are in fact even polynomial, and that the matrices $\pi(k, \xi)$ (for $k \in K_{P}$ ) are constant.

\subsection{Fourier transform on $L_{2}(\mathcal{H})$}

Let $V_{\xi}$ denote the representation space of $\pi(\xi), \xi \in \Xi$. Thus $V_{\xi}=$ $i\left(V_{\delta}\right)$ if $\xi=\left(P, \delta, t^{P}\right)$, and this vector space does not depend on the parameter $t^{P} \in T^{P}$. We denote by $\mathcal{V}_{\Xi}$ the trivial fibre bundle over $\Xi$ whose fibre at $\xi$ is $V_{\xi}$, thus

$$
\mathcal{V}_{\Xi}:=\cup_{(P, \delta)} \Xi_{P, \delta} \times i\left(V_{\delta}\right)
$$

where $\Xi_{P, \delta}$ denotes the component of $\Xi$ associated to $P \in \mathcal{P}$, and $\left(V_{\delta}, \delta\right) \in \Delta_{P}$, a complete set of representatives of the irreducible discrete series representations $\delta$ of $\mathcal{H}_{P}$. We denote by $\operatorname{End}\left(\mathcal{V}_{\Xi}\right)$ the endomorphism bundle of $\mathcal{V}_{\Xi}$, and by $\operatorname{Pol}\left(\Xi, \operatorname{End}\left(\mathcal{V}_{\Xi}\right)\right)$ the space of polynomial sections in this bundle. Similarly, let us introduce the space $\operatorname{Rat}^{r e g}\left(\Xi_{u}, \operatorname{End}\left(\mathcal{V}_{\Xi}\right)\right)$ of rational sections which are regular in a neighborhood of $\Xi_{u}$.

There is an action of $\mathcal{W}$ on $\operatorname{End}\left(\mathcal{V}_{\Xi}\right)$ as follows. If $(P, g) \in \mathcal{W}_{P}$ (the set of elements of $\mathcal{W}$ with source $P \in \mathcal{P}$ ) with $g=k \times n \in$ $K_{Q} \times W(P, Q), \xi \in \Xi_{P}$, and $A \in \operatorname{End}\left(V_{\xi}\right)$ we define $g(A):=\pi(g, \xi) \circ$ $A \circ \pi(g, \xi)^{-1} \in \operatorname{End}\left(V_{g(\xi)}\right)$. A section of $f$ of $\left.\operatorname{End}\left(\mathcal{V}_{\Xi}\right)\right)$ is called $\mathcal{W}$ equivariant if we have $f(\xi)=g^{-1}(f(g(\xi)))$ for all $\xi \in \Xi$ and $g \in \mathcal{W}_{\xi}$ (where $\mathcal{W}_{\xi}:=\mathcal{W}_{P}$ if $\xi=\left(P, \delta, t^{P}\right)$ ).

Definition 3.4. We define an averaging projection $p_{\mathcal{W}}$ onto the space of $\mathcal{W}$-equivariant sections by:

$$
p_{\mathcal{W}}(f)(\xi):=\left|\mathcal{W}_{\xi}\right|^{-1} \sum_{g \in \mathcal{W}_{\xi}} g^{-1}(f(g(\xi))) .
$$

Notice that this projection preserves the space $\operatorname{Rat}^{\text {reg }}\left(\Xi_{u}, \operatorname{End}\left(\mathcal{V}_{\Xi}\right)\right)$, but not the space $\operatorname{Pol}\left(\Xi, \operatorname{End}\left(\mathcal{V}_{\Xi}\right)\right)$. 
The Fourier transform $\mathcal{F}_{\mathcal{H}}$ on $\mathcal{H}$ is the following algebra homomorphism

$$
\begin{aligned}
\mathcal{F}_{\mathcal{H}}: \mathcal{H} & \rightarrow \operatorname{Pol}\left(\Xi_{u}, \operatorname{End}\left(\mathcal{V}_{\Xi}\right)\right)^{\mathcal{W}} \\
h & \rightarrow\{\xi \rightarrow \pi(\xi ; h)\}
\end{aligned}
$$

where $\operatorname{Pol}\left(\Xi, \operatorname{End}\left(\mathcal{V}_{\Xi}\right)\right)^{\mathcal{W}}$ denotes the space of $\mathcal{W}$-equivariant polynomial sections of $\left.\operatorname{End}\left(\mathcal{V}_{\Xi}\right)\right)$.

We will now describe a $\mathcal{W}$-invariant measure $\mu_{P l}$ on $\Xi_{u}$ whose push forward to $\mathcal{W} \backslash \Xi_{u}$ will be the Plancherel measure of $\mathcal{H}$ ([14, Theorem 4.43). Put $\xi=\left(P, \delta, t^{P}\right) \in \Xi_{u}$ and let $t=r_{P} t^{P}$. We write $d \xi:=$ $\left|K_{P, \delta}\right| d t^{P}$ where $d t^{P}$ denotes the normalized Haar measure of $T_{u}^{P}$ and where $K_{P, \delta}$ denotes the stabilizer of $\delta$ under the natural action of $K_{P}$ on $\Delta_{P}$. Let $\mathcal{K} \triangleleft \mathcal{W}$ denote the normal subgroupoid whose set of objects is $\mathcal{P}$, and with $\operatorname{Hom}_{\mathcal{K}}(P, Q)=\emptyset$ unless $P=Q$, in which case we have $\operatorname{Hom}_{\mathcal{K}}(P, P)=K_{P}$. Thus $\mathcal{W}_{P} / \mathcal{K}_{P}=\left\{w \in W_{0} \mid w(P) \subset F_{0}\right\}$. Let $\mu_{\mathcal{R}_{P}, P l}(\{\delta\})$ denote the Plancherel mass of $\delta$ with respect to $\mathcal{H}_{P}$ (see 14, Corollary 3.32 for a product formula for $\left.\mu_{\mathcal{R}_{P}, P l}(\{\delta\})\right)$. We now define the Plancherel measure $\mu_{P l}$ :

\section{Definition 3.5.}

$$
d \mu_{P l}(\xi):=q\left(w^{P}\right)^{-1}\left|\mathcal{W}_{P} / \mathcal{K}_{P}\right|^{-1} \mu_{\mathcal{R}_{P}, P l}(\{\delta\})|c(\xi)|^{-2} d \xi
$$

where $c(\xi)$ is the Macdonald c-function, see Definition 8.7 .

This measure is smooth on $\Xi_{u}$ (Proposition $8.8(\mathrm{v})$ ), and it is invariant for the action of $\mathcal{W}$ on $\Xi_{u}$, by Proposition 8.8 (ii).

With these notations we have:

Theorem 3.6. (14, Theorem 4.43)

(i) $\mathcal{F}_{\mathcal{H}}$ extends to an isometric isomorphism

$$
\mathcal{F}: L_{2}(\mathcal{H}) \rightarrow L_{2}\left(\Xi_{u}, \operatorname{End}\left(\mathcal{V}_{\Xi}\right), \mu_{P l}\right)^{\mathcal{W}},
$$

where the Hermitian inner product $(\cdot, \cdot)$ on $L_{2}\left(\Xi_{u}, \operatorname{End}\left(\mathcal{V}_{\Xi}\right), \mu_{P l}\right)^{\mathcal{W}}$ is defined by integrating the Hilbert-Schmidt form $(A, B):=$ $\operatorname{tr}\left(A^{*} B\right)$ in the fibres $\operatorname{End}\left(V_{\xi}\right)$ against the above measure $\mu_{P l}$ on the base space $\Xi_{u}$.

(ii) If $x \in C_{r}^{*}(\mathcal{H}) \subset L_{2}(\mathcal{H})$ then $\mathcal{F}(x) \in C\left(\Xi_{u}, \operatorname{End}\left(\mathcal{V}_{\Xi}\right)\right)^{\mathcal{W}}$.

(iii) Let $C_{r}^{*}(\mathcal{H})^{o}$ denote the opposite $C^{*}$-algebra of $C_{r}^{*}(\mathcal{H})$. Let $(x, y) \in C_{r}^{*}(\mathcal{H}) \times C_{r}^{*}(\mathcal{H})^{o}$ act on $L_{2}(\mathcal{H})$ via the regular representation $\lambda(x) \times \rho(y)$, and on $L_{2}\left(\Xi_{u}, \operatorname{End}\left(\mathcal{V}_{\Xi}\right), \mu_{P l}\right)^{\mathcal{W}}$ through fibrewise multiplication from the left with $\mathcal{F}(x)$ and from the right with $\mathcal{F}(y)$. Then $\mathcal{F}$ intertwines these representations of $C_{r}^{*}(\mathcal{H}) \times C_{r}^{*}(\mathcal{H})^{o}$. 
Proof. As to (ii), first recall that according to Equation $(3.9), \mathcal{F}_{\mathcal{H}}(\mathcal{H}) \subset$ $\operatorname{Pol}\left(\Xi_{u}, \operatorname{End}\left(\mathcal{V}_{\Xi}\right)\right)^{\mathcal{W}}$. By $([14$, Theorem 4.43(iii) $)$ one easily deduces that $\|h\|_{o}=\left\|\mathcal{F}_{\mathcal{H}}(h)\right\|_{\text {sup }}$ for all $h \in \mathcal{H}$, where $\|\sigma\|_{\text {sup }}:=\sup _{\xi \in \Xi_{u}}\|\sigma(\xi)\|_{o}$ (where $\|\sigma(\xi)\|_{o}$ denotes the operatornorm of $\sigma(\xi) \in \operatorname{End}\left(V_{\xi}\right)$ ). Hence $\mathcal{F}\left(C_{r}^{*}(\mathcal{H})\right) \subset C\left(\Xi_{u}, \operatorname{End}\left(\mathcal{V}_{\Xi}\right)\right)^{\mathcal{W}}$.

Now (iii) follows from (ii) and ([14, Theorem 4.43(iii)).

The following easy corollary is important in the sequel:

Corollary 3.7. (14, Theorem 4.45) The averaging operator $p_{\mathcal{W}} d e-$ fines an orthogonal projection onto the space of $\mathcal{W}$-equivariant sections in $L_{2}\left(\Xi_{u}, \operatorname{End}\left(\mathcal{V}_{\Xi}\right), \mu_{P l}\right)$. Moreover, if

$$
\mathcal{J}: L_{2}\left(\Xi_{u}, \operatorname{End}\left(\mathcal{V}_{\Xi}\right), \mu_{P l}\right) \rightarrow L_{2}(\mathcal{H})
$$

denotes the adjoint of $\mathcal{F}$ (the wave packet operator), then $\mathcal{J} \mathcal{F}=\mathrm{id}$ and $\mathcal{F} \mathcal{J}=p_{\mathcal{W}}$.

Proof. Theorem 3.6 implies that $\mathcal{J} \mathcal{F}:=$ id and that $\mathcal{F} \mathcal{J}$ is equal to the orthogonal projection onto the space of $\mathcal{W}$-equivariant $L_{2}$-sections of $\operatorname{End}\left(\mathcal{V}_{\Xi}\right)$.

On the other hand, since the action of $\mathcal{W}$ on $\operatorname{End}\left(\mathcal{V}_{\Xi}\right)$ is defined in terms of invertible smooth matrices (cf. Theorem [3.3), $p_{\mathcal{W}}$ preserves the space of $L_{2}$-sections. By the $\mathcal{W}$-invariance of $\mu_{P l}$, the projection $p_{\mathcal{W}}$ on $L_{2}\left(\Xi_{u}, \operatorname{End}\left(\mathcal{V}_{\Xi}\right), \mu_{P l}\right)$ is in fact an orthogonal projection. This finishes the proof.

\section{Main Theorem and its applications}

The space of smooth section of the trivial bundle $\operatorname{End}\left(\mathcal{V}_{\Xi}\right)$ on $\Xi_{u}$ will be denoted by $C^{\infty}\left(\Xi_{u}, \operatorname{End}\left(\mathcal{V}_{\Xi}\right)\right)$. We equip this vector space with its usual Fréchet topology. The collection of semi-norms inducing the topology is of the form $p(\sigma):=\sup _{\xi \in \Xi_{u}}\|D \sigma(\xi)\|_{o}$, where $D$ is a constant coefficient differential operator on $\Xi_{u}$ (i.e. one such operator for each connected component of $\Xi_{u}$ ), acting entrywise on the section $\sigma$ of the trivial bundle $\operatorname{End}\left(\mathcal{V}_{\Xi}\right)$, and where $\|\cdot\|_{o}$ denotes the operatornorm. It is obvious from the product rule for differentiation that $C^{\infty}\left(\Xi_{u}, \operatorname{End}\left(\mathcal{V}_{\Xi}\right)\right)$ is a Fréchet algebra.

The projection $p_{\mathcal{W}}$ is continuous on $C^{\infty}\left(\Xi_{u}, \operatorname{End}\left(\mathcal{V}_{\Xi}\right)\right)$, since it is defined in terms of the action of $\mathcal{W}$ on $\Xi_{u}$, and conjugations with invertible smooth matrices. Thus the subalgebra $C^{\infty}\left(\Xi_{u}, \operatorname{End}\left(\mathcal{V}_{\Xi}\right)\right)^{\mathcal{W}}$ of $\mathcal{W}$-equivariant sections is a closed subalgebra.

We now define the vector space 


\section{Definition 4.1.}

$$
\mathcal{C}\left(\Xi_{u}, \operatorname{End}\left(V_{\Xi}\right)\right):=c C^{\infty}\left(\Xi_{u}, \operatorname{End}\left(\mathcal{V}_{\Xi}\right)\right),
$$

where $c$ denotes the c-function of Definition 8.7 on $\Xi_{u}$. We equip $\mathcal{C}\left(\Xi_{u}, \operatorname{End}\left(V_{\Xi}\right)\right)$ with the Fréchet space topology of $C^{\infty}\left(\Xi_{u}, \operatorname{End}\left(V_{\Xi}\right)\right)$ via the linear isomorphism $C^{\infty}\left(\Xi_{u}, \operatorname{End}\left(V_{\Xi}\right)\right) \rightarrow \mathcal{C}\left(\Xi_{u}\right.$, End $\left.\left(V_{\Xi}\right)\right)$ defined by $\sigma \rightarrow c \sigma$.

Lemma 4.2. The complex vector space $\mathcal{C}\left(\Xi_{u}, \operatorname{End}\left(V_{\Xi}\right)\right)$ is closed for taking (fibrewise) adjoints, and

$$
\mathcal{C}\left(\Xi_{u}, \operatorname{End}\left(V_{\Xi}\right)\right) \subset L_{2}\left(\Xi_{u}, \operatorname{End}\left(V_{\Xi}\right), \mu_{P l}\right) .
$$

Moreover,

$$
C^{\infty}\left(\Xi_{u}, \operatorname{End}\left(V_{\Xi}\right)\right) \subset \mathcal{C}\left(\Xi_{u}, \operatorname{End}\left(V_{\Xi}\right)\right)
$$

is a closed subspace.

Proof. It is closed for taking adjoints by Proposition 8.8(iv) (applied to $\left.d=w^{P} \in W\left(P, P^{\prime}\right)\right)$, and it is a subspace of $L_{2}\left(\Xi_{u}\right.$, End $\left.\left(V_{\Xi}\right), \mu_{P l}\right)$ by Proposition 8.8(i). The last assertion follows from Proposition 8.8(v).

Now we are prepared to formulate the main theorem of this paper.

Theorem 4.3. The Fourier transform restricts to an isomorphism of Fréchet algebras

$$
\mathcal{F}_{\mathcal{S}}: \mathcal{S} \rightarrow C^{\infty}\left(\Xi_{u}, \operatorname{End}\left(\mathcal{V}_{\Xi}\right)\right)^{\mathcal{W}}
$$

The wave packet operator $\mathcal{J}$ restricts to a surjective continuous map

$$
\mathcal{J}_{\mathcal{C}}: \mathcal{C}\left(\Xi_{u}, \operatorname{End}\left(\mathcal{V}_{\Xi}\right)\right) \rightarrow \mathcal{S}
$$

We have $\mathcal{J}_{\mathcal{C}} \mathcal{F}_{\mathcal{S}}=\mathrm{id}_{\mathcal{S}}$, and $\mathcal{F}_{\mathcal{S}} \mathcal{J}_{\mathcal{C}}=p_{\mathcal{W}, \mathcal{C}}$ (the restriction of $p_{\mathcal{W}}$ to $\left.\mathcal{C}\left(\Xi_{u}, \operatorname{End}\left(\mathcal{V}_{\Xi}\right)\right)\right)$. In particular, the map $p_{\mathcal{W}, \mathcal{C}}$ is a continuous projection of $\mathcal{C}\left(\Xi_{u}, \operatorname{End}\left(V_{\Xi}\right)\right)$ onto $C^{\infty}\left(\Xi_{u}, \operatorname{End}\left(V_{\Xi}\right)\right)^{\mathcal{W}}$.

\subsection{Applications of the Main Theorem}

Before we embark on its proof we discuss some immediate consequences of the Main Theorem.

Corollary 4.4. (Harish-Chandra's completeness Theorem, cf. [8], and 10, Theorem 14.31) Let $\xi \in \Xi_{u}$. The complex linear span $C_{\xi}$ of the set of operators $\left\{\pi(g, \xi) \mid g \in \operatorname{End}_{\mathcal{W}_{\Xi}}\left(V_{\xi}\right)\right\}$ is a unital, involutive subalgebra of $\operatorname{End}\left(V_{\xi}\right)$. For all $\xi \in \Xi_{u}$, the centralizer algebra $\pi(\xi, \mathcal{H})^{\prime}$ is equal to $C_{\xi}$. 
Proof. Let $\xi=\left(P, \delta, t^{P}\right)$ and denote by $C_{\xi} \subset \operatorname{End}\left(i\left(V_{\delta}\right)\right)$ the complex linear span of the set of operators $\left\{\pi(g, \xi) \mid g \in \operatorname{End}_{\mathcal{W} \Xi}\left(V_{\xi}\right)\right\}$. By Theorem 3.3. $C_{\xi}$ is an involutive (i.e. $*$-invariant), unital subalgebra of $\operatorname{End}\left(i\left(V_{\delta}\right)\right)$. Theorem 4.3 implies that $\pi(\xi, \mathcal{H})=C_{\xi}^{\prime}$. The Bicommutant Theorem therefore implies that $C_{\xi}=\pi(\xi, \mathcal{H})^{\prime}$.

Corollary 4.5. The center $\mathcal{Z}_{\mathcal{S}}$ of $\mathcal{S}$ is, via the Fourier Transform $\mathcal{F}_{\mathcal{S}}$, isomorphic to the algebra $C^{\infty}\left(\Xi_{u}\right)^{\mathcal{W}}$.

Proof. The algebra of scalar sections of $C^{\infty}\left(\Xi_{u}, \operatorname{End}\left(\mathcal{V}_{\Xi_{u}}\right)\right)^{\mathcal{W}}$ is isomorphic to $C^{\infty}\left(\Xi_{u}\right)^{\mathcal{W}}$, and is contained in $\mathcal{F}_{\mathcal{S}}\left(\mathcal{Z}_{\mathcal{S}}\right)$ by Theorem 4.3. To show the equality, observe that Corollary 4.4 implies that an element of $\mathcal{F}_{\mathcal{S}}\left(\mathcal{Z}_{\mathcal{S}}\right)$ is scalar at all fibers $\operatorname{End}\left(V_{\xi}\right)$ with $\xi \in \Xi_{u}$ generic (since $\operatorname{End}_{\mathcal{W} \Xi}\left(V_{\xi}\right)=\mathbb{C}$ in this case). By the density of the set of generic points in $\Xi_{u}$ we obtain the desired equality.

Notice that $\mathcal{Z}_{\mathcal{S}}$ is in general larger than the closure in $\mathcal{S}$ of the center $\mathcal{Z}$ of $\mathcal{H}$.

Corollary 4.6. (Langlands' disjointness Theorem, cf. [10], Theorem 14.90) Let $\xi, \xi^{\prime} \in \Xi_{u}$. If $\pi(\xi)$ and $\pi\left(\xi^{\prime}\right)$ are not disjoint, then the objects $\xi$, $\xi^{\prime}$ of $\mathcal{W}_{\Xi_{u}}$ are isomorphic (and thus, $\pi(\xi)$ and $\pi\left(\xi^{\prime}\right)$ are actually equivalent).

Proof. Corollary 4.5 implies that $\mathcal{Z}_{\mathcal{S}}$ separates the $\mathcal{W}$-orbits of $\Xi_{u}$. Whence the result.

Corollary 4.7. The Fourier Transform $\mathcal{F}$ restricts to a $C^{*}$-algebra isomorphism

$$
\mathcal{F}_{C}: C_{r}^{*}(\mathcal{H}) \rightarrow C\left(\Xi_{u}, \operatorname{End}\left(\mathcal{V}_{\Xi}\right)\right)^{\mathcal{W}}
$$

where $C_{r}^{*}(\mathcal{H})$ denotes the reduced $C^{*}$-algebra of $\mathcal{H}$ (cf. 14, Definition 2.4).

Proof. By Theorem [3.6 the restriction of $\mathcal{F}$ to $C_{r}^{*}(\mathcal{H})$ is an algebra homomorphism. It is a homomorphism of involutive algebras since $\pi\left(\xi ; x^{*}\right)=\pi(\xi ; x)^{*}($ cf. Subsection 3.1) $)$.

The reduced $C^{*}$-algebra $C_{r}^{*}(\mathcal{H})$ of $\mathcal{H}$ is defined in 14] as the norm closure of $\lambda(\mathcal{H}) \subset B\left(L_{2}(\mathcal{H})\right)$. By Theorem 3.6. the norm $\|x\|_{o}$ of $C_{r}^{*}(\mathcal{H})$ is equal to the supremum norm $\|\mathcal{F}(x)\|_{\text {sup }}$ of the $\mathcal{W}$-invariant continuous function $\xi \rightarrow\|\pi(\xi ; x)\|_{o}$ on $\Xi_{u}$ (where $\|\pi(\xi ; h)\|_{o}$ denotes the operator norm for operators on the finite dimensional Hilbert space $V_{\xi}=i\left(V_{\delta}\right)$ ). Notice that, by the regularity of the standard intertwining operators, the projection operator $p_{\mathcal{W}}$ restricts to a continuous projection on the space of continuous sections of $\operatorname{End}\left(\mathcal{V}_{\Xi}\right)$. 
By Theorem 4.3, the closure of $\mathcal{F}(\mathcal{S})$ with respect to $\|\cdot\|_{\text {sup }}$ is equal to $C\left(\Xi_{u}, \operatorname{End}\left(\mathcal{V}_{\Xi}\right)\right)^{\mathcal{W}}$. In view of Theorem 3.6 (ii) this finishes the proof.

Corollary 4.8. The set of minimal central idempotents of $C_{r}^{*}(\mathcal{H})$ is parameterized by the (finite) set of $\mathcal{W}$-orbits of pairs $(P, \delta)$ with $P \in \mathcal{P}$ and $\delta \in \Delta_{P}$. The central idempotents $e_{(P, \delta)}$ are elements of $\mathcal{S}$.

Proof. This is immediate from Theorem 4.3 and Corollary 4.7.

Corollary 4.9. The dense subalgebra $\mathcal{S} \subset C_{r}^{*}(\mathcal{H})$ is closed for holomorphic functional calculus.

Proof. According to a well known criterium for closedness under holomorphic functional calculus, we need to check that if $a \in \mathcal{S}$ is invertible in $C_{r}^{*}(\mathcal{H})$, then $a^{-1} \in \mathcal{S}$. This is obvious from Theorem 4.3 and 4.7

\section{Constant terms of matrix coefficients of $\pi(\xi)$}

In the remainder of this paper we will prove the Main Theorem Theorem 4.3. A main tool is the notion of the constant term $f^{P}$ of a function $f \in \mathbb{A}^{t e m p}$ with respect to a standard parabolic subset $P \in \mathcal{P}$.

\subsection{Definition of the constant terms of $f \in \mathbb{A}^{\text {temp }}$}

It this section we define the constant term of a tempered finite functional $f \in \mathbb{A}^{\text {temp }}$ along a standard parabolic subalgebra $\mathcal{H}^{P}$ of $\mathcal{H}$. Recall the notion of exponents 2.10 and the Casselman criteria for tempered finite functionals.

Definition 5.1. Let $P \subset F_{0}$, and $R_{P}$ the standard parabolic subsystem with that subset. For real characters $t_{1}, t_{2}$ on $X$ we say that $t_{1} \leq_{P} t_{2}$ if and only if $t_{1}(x) \leq t_{2}(x)$ for all $x \in X^{P,+}:=\{x \in X \mid \forall \alpha \in$ $\left.P:\left\langle x, \alpha^{\vee}\right\rangle \geq 0\right\}$. In other words, $t_{1} \leq_{P} t_{2}$ iff both $t_{1} \leq_{F_{0}} t_{2}$ and $\left.t_{1}\right|_{X \cap P^{\perp}}=\left.t_{2}\right|_{X \cap P^{\perp}}$.

Thus $t_{1} \leq_{P} t_{2}$ iff $t_{1} t_{2}^{-1}=\prod_{\alpha \in P}\left(d_{\alpha} \otimes \alpha^{\vee}\right)$ with $0<d_{\alpha} \leq 1$, where $d \otimes \alpha^{\vee} \in T_{r s}$ is the real character defined by $d \otimes \alpha^{\vee}(x)=d^{\left\langle x, \alpha^{\vee}\right\rangle}$.

Definition 5.2. (Constant term) Let $P \in \mathcal{P}$ and $f \in \mathbb{A}^{\text {temp }}$. Then we define the constant term of $f$ along $P$ by

$$
f^{P}(h):=\sum_{t \in \epsilon:|t| \leq \leq_{P} 1} f_{t}(h) .
$$


where (in the notation of Corollary 2.14) $f_{t}(h):=E_{t}^{f}(h, 0)$, and the coefficients $E_{t}^{f}$ and the set $\epsilon$ are defined by the expansion 2.15. We say that an exponent $t \in \epsilon$ of $f$ is $P$-tempered if it satisfies the condition $|t| \leq_{P} 1$.

The notion of cuspidality (cf. Definition 2.17) can now be reformulated as follows:

Corollary 5.3. Let $f \in \mathbb{A}^{\text {temp }}$. Then $f \in \mathbb{A}^{\text {cusp }}$ iff $f^{P}=0$ for every proper $P \in \mathcal{P}$.

Observe the following elementary properties of the constant term:

Corollary 5.4. $\quad$ (i) $f^{P} \in \mathbb{A}^{\text {temp }}$.

(ii) $L_{x}$ commutes with $f \rightarrow f^{P}$ if $x \in \mathcal{H}^{P}$.

(iii) $R_{y}$ commutes with $f \rightarrow f^{P}$ for all $y \in \mathcal{H}$.

(iv) $f^{P} \in L_{X}(f)=f \cdot \mathcal{A} \subset f \cdot \mathcal{H}$.

The projection of $f$ to $f^{P}$ can be made explicit using an idempotent $e^{P}$ in a formal completion of $\mathcal{A} \subset \mathcal{H}$. Such completions were introduced and studied by Lusztig [11] (cf. Subsection [2.12). This will be applied to the case were $f$ is a matrix coefficient of a parabolically induced representation in the next subsection.

\subsection{Constant terms of coefficients of $\pi(\xi)$ for $\xi \in \Xi_{u}$ generic}

In this subsection we assume that $\xi$ is generic unless stated otherwise

We will now discuss the constant terms of a matrix coefficient of $\pi=\pi(\xi)$ in the case where $\xi=\left(P, \delta, t^{P}\right) \in \Xi_{u}$ is generic. Choose $r_{P} \in T_{P}$ such that $W_{P} r_{P}$ is the central character of $\delta$. We thus assume

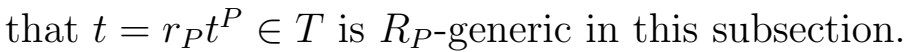

Let $a, b \in i\left(V_{\delta}\right)$, and denote by $f=f_{a, b}=f_{a, b}(\xi)$ the matrix coefficient defined by $f(h)=\langle a, \pi(\xi ; h)(b)\rangle$. By [14, Lemma 2.20 and Proposition 4.20, we have: If $t^{P} \in T_{u}^{P}$ then $f_{a, b} \in \mathbb{A}^{\text {temp }}$ for all $a, b \in \mathcal{H}\left(W^{P}\right) \otimes V_{\delta}$. More precisely:

Proposition 5.5. The exponents of $f$ are of the form $w t^{\prime}$ where $w$ runs over the set $W^{P}$ and where $t^{\prime}$ runs over the set of weights of $\delta_{t^{P}}$, thus $t^{P}$ times the set of $X_{P}$-weights of $\delta$.

Now let $Q \subset F_{0}$ be another standard parabolic. By proof of [14], Proposition 4.20 we deduce:

Proposition 5.6. Let $w \in W^{P}$ and let $u \in W_{P}$ such that wut is an exponent of $f$. If $w u|t| \leq_{Q} 1$, then $w(P) \subset R_{Q,+}$. 
Proof. The equivalence class $\varpi$ of $t=r_{P} t^{P}$ is equal to $W_{P} t$ (since we assume genericity). If $w u t$ is an exponent then $w u t=w^{\prime} t^{\prime}$ with $t^{\prime}$ an $X$-weight of $\delta_{t^{P}}$ and $w^{\prime} \in W^{P}$. Thus $t^{\prime}$ and $u t$ are both in $\varpi$, the equivalence class of $t$. Hence by genericity, $w^{\prime}=w$ and thus $u t=t^{\prime}$, a weight of $\delta_{t^{P}}$. But $\delta$ is discrete series for $\mathcal{H}_{P}$, hence $|u t|=\prod_{\alpha \in P} d_{\alpha} \otimes \alpha^{\vee}$ with all $0<d_{\alpha}<1$. Thus $w u|t|=\prod_{\alpha \in P} d_{\alpha} \otimes w\left(\alpha^{\vee}\right) \leq_{Q} 1$ implies (since for all $\left.\alpha \in P: w\left(\alpha^{\vee}\right) \in R_{0,+}^{\vee}\right)$ that $w(P) \subset R_{Q,+}$.

Corollary 5.7. Recall that the equivalence classes in $W_{0} t$ are of the form $w \varpi$ with $\varpi=W_{P}$ and $w \in W^{P}$. If an exponent $w t^{\prime}$ of $f$ (with $w \in W^{P}$ and $t^{\prime}$ a weight of $\left.\delta_{t^{P}}\right)$ is Q-tempered, then all exponents of $f$ in its class $w \varpi$ are $Q$-tempered. The class $w \varpi\left(w \in W^{P}\right)$ is $Q$-tempered if and only if $w(P) \subset R_{Q,+}$.

Proof. Since $w(P) \subset R_{Q,+}$ we have $w W_{P} w^{-1} \subset W_{Q}$. Hence $w \varpi \subset$ $W_{Q} w t$, so that all elements of $w \varpi$ have the same restriction 1 to $X \cap Q^{\perp}$.

Now we will express the constant term of a matrix coefficient of $\pi(\xi)$ in terms of the idempotents $e_{\varpi}$ of the completion $\overline{\mathcal{H}}_{t}$. Recall the material of Subsection 2.12.

We will use the analog of Lusztig's first reduction theorem (2.18) for $\overline{\mathcal{H}}_{t}$, in combination with the results in ([14, Section 4.3) on the Hilbert algebra structure of $\overline{\mathcal{H}^{t}}$, the quotient of $\mathcal{H}^{t}$ by the radical of the positive semi-definite Hermitian pairing $(x, y)_{t}:=\chi_{t}\left(x^{*} y\right)$, in order to express and study the constant term.

Proposition 5.8. We have that

$$
f^{Q}(h)=\sum_{w \in W^{P}: w(P) \subset R_{Q,+}} f\left(e_{w \varpi} h\right)
$$

Proof. Let us denote by $J_{w}$ the ideal in $\mathcal{A}^{W_{w(P)}}$ of elements in this ring vanishing at $w \varpi$. Clearly $\mathcal{I}_{t} \subset J_{w}$ for all $w$. By some elementary algebra (similar to proof of Prop 2.24(4) in [13]) we see that for every $x \in J_{w}$ and $k \in \mathbb{N}$ there exist a $\bar{x} \in \mathcal{I}_{t}$ and a unit $e$ in $\overline{\mathcal{A}}_{m_{w}}$ such that

$$
e x \in \bar{x}+m_{w}^{k}
$$

where $m_{w}$ denotes the ideal of all functions in $\mathcal{A}$ which vanish at the points of $w \varpi$. (To be sure, we construct $\bar{x}$ by first adding an element $u \in J_{w}^{k}$ such that $x+u$ is nonzero at the other classes $w^{\prime} \varpi$ with $w^{\prime} \in$ $W^{P}, w^{\prime} \neq w$. Take $\bar{x}$ equal to the product of the translates $(x+u)^{w}$ where $w$ runs over the set of left cosets $W_{0} / W_{w(P)}$. Let $e$ be equal to the product of these factors $(x+u)^{w}$ where $w$ runs over the set of left 
cosets $W_{0} / W_{w(P)}$ with $\left.w \neq W_{w(P)}\right)$. Let $M$ be the ideal of functions in $\mathcal{A}$ vanishing at $W_{0} t$. Then $M=\prod m_{w}$ and by genericity the ideals $m_{w}$ are relatively prime. So $\overline{\mathcal{A}}_{M}=\oplus \overline{\mathcal{A}}_{m_{w}}$ by the Chinese remainder theorem. Then $e_{w \varpi}$ is the unit of the summand $\overline{\mathcal{A}}_{m_{w}}$. Let $\bar{e}_{w \varpi}$ be the unit of $R:=\overline{\mathcal{A}}_{m_{w}} / \mathcal{I}_{t} \overline{\mathcal{A}}_{m_{w}}$ (the canonical image of $e_{w \varpi} \in \overline{\mathcal{A}}_{m_{w}}$ ). Note that $R$ is finite dimensional over $\mathbb{C}$, and thus $R$ is Artinian. By definition of $m_{w}, m_{w} \bar{e}_{w \varpi}$ is contained in all the maximal ideals of $R$. Hence $m_{w} \bar{e}_{w \varpi}$ is contained in the intersection of the maximal ideals of $R$, which is nilpotent in $R$ (see the proof of Theorem 3.2 of [12]). In particular, for sufficiently large $k, m_{w}^{k} e_{w \varpi} \subset \mathcal{I}_{t} \overline{\mathcal{A}}_{m_{w}}$, whence

$$
x e_{w \varpi} \in \mathcal{I}_{t} \overline{\mathcal{A}}_{m_{w}} \text {. }
$$

But then the right hand side is in the kernel of $\pi$, thus we conclude that $J_{w} e_{w \varpi}$ is in the kernel of $\pi$. In particular, the element (for any $z \in X) \Theta_{z}:=\prod_{y \in W_{w(P)}}\left((w t)(z)^{-1} \theta_{y}-1\right) \in J_{w}$ acts by zero on the finite dimensional space of left $\mathcal{A}$-translates of $h \rightarrow f\left(e_{w \varpi} h\right)$. Thus the exponents of $f \rightarrow f\left(e_{w \varpi} h\right)$ are contained in $w \varpi$.

We obviously have

$$
f(h)=\sum_{w \in W^{P}} f\left(e_{w \varpi} h\right)
$$

(splitting of 1 according the decomposition of $\overline{\mathcal{A}}_{M}$ ). By the results in this paragraph, an exponent of $h \rightarrow f\left(e_{w \varpi} h\right)$ is $Q$-tempered iff all exponents of this term are $Q$-tempered iff $w(P) \subset R_{Q,+}$. Hence the result.

Corollary 5.9. The constituents $f\left(e_{w \varpi} h\right)$ depend on the induction parameter $t^{P}$ as a rational function.

Proof. In the proof of Corollary 5.8 we can equally well work over the field $K$ of rational functions on $T^{P}$ instead of $\mathbb{C}$. Then $\bar{e}_{w \varpi} \in$ $\overline{\mathcal{A}}(K)_{M} / \mathcal{I}_{t} \overline{\mathcal{A}}(K)_{M}=\mathcal{A}(K) / \mathcal{I}_{t} \mathcal{A}(K)$. Hence the result.

\subsection{Some results for Weyl groups}

We want to work with standard parabolics only, and $w(P) \subset R_{Q,+}$ does not need to be standard. We resolve this by combining terms according to left $W_{Q}$ cosets. We use the following results (see [4], Section 2.7).

Proposition 5.10. Let $P, Q \in \mathcal{P}$. The set $D^{Q, P}:=\left(W^{Q}\right)^{-1} \cap W^{P}$ intersects every double coset $W_{Q} w W_{P}$ in precisely one element $d=$ $d(w)$, which is the unique element of shortest length of the double coset. 
Proposition 5.11. (Kilmoyer) Let $d \in D^{Q, P}$. Then $W_{Q} \cap W_{d(P)}$ is the standard parabolic subgroup of $W_{0}$ corresponding to the subset $L=$ $Q \cap d(P)$.

Let $t \in r_{P} T^{P}$ be $W_{P}$-generic as before, where $W_{P} r_{P} \subset T_{P}$ is the central character of a discrete series representation $\delta$ of $\mathcal{H}_{P}$. Let $\varpi=$ $W_{P} t$ be the equivalence class of $t$.

Corollary 5.12. (i) Let $w \in W^{P}$ be such that w $\varpi$ is $Q$-tempered. Then $w(P) \subset R_{Q,+}$. We can write $w=u d$ with $d=d(w) \in$ $D^{Q, P}$ and $u \in W_{Q}$. Then $d(P) \subset Q$, and $u \in W_{Q}^{d(P)}$. Conversely, if $d \in D^{Q, P}$ is such that $d(P) \subset Q$, then for all $u \in W_{Q}^{d(P)}$ we have $|u d(\varpi)| \leq_{Q} 1$ (in other words, is $Q$ tempered).

(ii) The classes $\varpi_{u, d}=: u d(\varpi)$ with $d \in D^{Q, P}$ such that $d(P) \subset Q$ and $u \in W_{Q}^{d(P)}$, are mutually disjoint.

Proof. (i) According to a result of Howlett (cf. 44, Proposition 2.7.5), we can uniquely decompose $w$ as a product of the form $w=u d v$ with $d=d(w) \in D^{Q, P}, u \in W_{Q} \cap W^{L}$ (with $L=Q \cap d(P)$ ), and $v \in W_{P}$. In fact $v=e$, since otherwise there would exist a $\alpha \in R_{P,+}$ with $v(\alpha)=-\alpha_{p} \in P$. But then $u d\left(\alpha_{p}\right)<0$, which implies according to [4], Lemma 2.7.1 that $d\left(\alpha_{p}\right)=\alpha_{q} \in L$. Hence $u\left(\alpha_{q}\right)<0$, which contradicts the assumption $u \in W_{Q} \cap W^{L}$. Thus we have $d(P)=u^{-1} w(P) \subset R_{Q,+}$, whence $W_{d(P)} \subset W_{Q}$. By Kilmoyer's result it now follows that $W_{d(P)}=$ $W_{Q \cap d(P)}$. Hence $d(P) \subset Q$ and $L=d(P)$. The converse is clear.

(ii) Suppose that $\varpi_{u, d} \cap \varpi_{u^{\prime}, d^{\prime}} \neq \emptyset$. The Weyl group $W_{0}$ permutes equivalence classes, thus this implies that $(u d)^{-1} u^{\prime} d^{\prime}(t) \in \varpi$. Since $t$ is generic, there exists a $v \in W_{P}$ such that $u^{\prime} d^{\prime}=u d v$. By Howlett's result [4, Proposition 2.7.5 this implies that $v=1, u=u^{\prime}$ and $d=d^{\prime}$.

Corollary 5.13. For all $d \in D^{Q, P}$ with $d(P) \subset Q$ we write

$$
e_{W_{Q} d \varpi}=\sum_{u \in W_{Q}^{d(P)}} e_{u d \varpi}
$$

This is a collection of orthogonal idempotents of $\overline{\mathcal{H}}_{t}$. The constant term of $f=f_{a, b}(\xi)$ equals

$$
f^{Q}(h)=\sum_{d \in D^{Q, P}: d(P) \subset Q} f^{d}(h),
$$


where we define $f^{d}(h):=f\left(e_{W_{Q} d \varpi} h\right)$. This is the contribution to the constant term $f^{Q}$ of $f$ whose exponents have the same restriction to $X \cap Q^{\perp}$ as $d(t)$.

\subsection{The singularities of $f^{d}$}

In this section we take the formulae of Corollary 5.13 as a definition of $f^{Q}$ and $f^{d}$, even when $t^{P} \in T^{P}$ is not in $T_{u}^{P}$.

We will now bound the possible singularities of the individual contributions $f^{d}$ to $f^{Q}$, viewed as functions of $t^{P} \in T^{P}$. We have seen in Corollary 5.9 that $f^{d}$ extends to a rational function of $\xi \in \Xi$. To stress this dependence we sometimes write $f^{d}(\xi, h)$. We write $\xi=\left(P, \delta, t^{P}\right)$ and put $t=t(\xi)=r_{P} t^{P}$, where $r_{P} \in T_{P}$ is such that $W_{P} r_{P}$ is equal to the central character of $\delta$.

Lemma 5.14. Let $P, Q \in \mathcal{P}$ and let $d \in D^{Q, P}$ be such that $d(P) \subset Q$. Let $h, h^{\prime} \in \overline{\mathcal{H}}_{t}$. Then

$$
f_{a, b}^{d}\left(\xi ; h h^{\prime}\right)=f_{a, \pi\left(\xi ; h^{\prime}\right)(b)}^{d}(\xi ; h) .
$$

Proof. This follows immediately from Corollary 5.4.

Lemma 5.15. As in Lemma 5.14. Let $g \in \mathcal{W}_{P}$ and put $P^{\prime}=g(P)$. According to Corollary 5.12 we can write $d g^{-1}=u^{\prime} d^{\prime}$ with $d^{\prime} \in D^{Q, P^{\prime}}$ and $u^{\prime} \in W_{Q}^{P^{\prime}}$. We put $t^{\prime}=g(t)$ and $\varpi^{\prime}=W_{P^{\prime}} t^{\prime}=g(\varpi)$, so that $e_{W_{Q} d \varpi}=e_{W_{Q} d^{\prime} \varpi^{\prime}}$. With these notations we have the following equality of rational functions of $\xi$ :

$$
f_{a, b}^{d}(\xi ; h)=f_{\pi\left(g, \bar{\xi}^{-1}\right)(a), \pi(g, \xi)(b)}^{d^{\prime}}(g(\xi) ; h),
$$

where $\bar{\xi}^{-1}:=\left(P, \delta, \bar{t}^{-1}\right)$.

Proof. This equation follows from the special case $\xi \in \Xi_{P, \delta, u}$ because the left hand side and the right hand side are obviously rational functions of $\xi$. In this special case the equation simply expresses the unitarity of the intertwiners (cf. Theorem 3.3).

Lemma 5.16. Let $P, Q \in \mathcal{P}$. Then $\mathcal{H}$ has the following direct sum decomposition in left $\mathcal{H}^{Q}$-right $\mathcal{H}\left(W_{P}\right)$-submodules:

$$
\mathcal{H}=\bigoplus_{d \in D^{Q, P}} \mathcal{H}_{Q, P}(d)
$$

where $\mathcal{H}_{Q, P}(d):=\mathcal{H}^{Q} N_{d} \mathcal{H}\left(W_{P}\right)$. 
Proof. Using the Bernstein presentation of $\mathcal{H}^{Q}$ and the definition of the multiplication in $\mathcal{H}\left(W_{0}\right)$ we easily see that

$$
\mathcal{H}_{Q, P}(d)=\bigoplus_{w \in W_{Q} d W_{P}} \mathcal{A} N_{w}
$$

The result thus follows from the Bernstein presentation of $\mathcal{H}$.

After these preparations we will now concentrate on an important special case.

Definition 5.17. Let $\pi_{Q, P}^{d}: \mathcal{H} \rightarrow \mathcal{H}_{Q, P}(d)$ denote the projection according to the above direct sum decomposition. Given $Q \in \mathcal{P}$, denote by $w^{Q}=w_{0} w_{Q}$ the longest element of $W^{Q}$, and by $Q^{\prime}=w^{Q}(Q)=$ $-w_{0}(Q) \in \mathcal{P}$. Then $w^{Q^{\prime}}=\left(w^{Q}\right)^{-1} \in D^{Q, Q^{\prime}}$, and

$$
\mathcal{H}_{Q, Q^{\prime}}\left(w^{Q^{\prime}}\right)=\mathcal{H}^{Q} N_{w^{Q^{\prime}}}=\mathcal{A} N_{w^{Q^{\prime}}} \mathcal{H}\left(W_{Q^{\prime}}\right) \text {. }
$$

Let $p_{Q}: \mathcal{H} \rightarrow \mathcal{H}^{Q}$ be the left $\mathcal{H}^{Q}$-module map defined by

$$
p_{Q}(h):=\pi_{Q, Q^{\prime}}^{w^{Q^{\prime}}}(h) N_{w^{Q^{\prime}}}^{-1}
$$

(Observe that this map indeed has values in $\mathcal{H}^{Q}$ by (5.4)).

In (15.5) we have used that $N_{w^{Q^{\prime}}} N_{w^{\prime}}=N_{w} N_{w^{Q^{\prime}}}$ if $w \in W_{Q}$.

Theorem 5.18. Let $P, Q \in \mathcal{P}$ be such that $P \subset Q$. We put $P^{\prime}:=$ $w^{Q}(P) \subset Q^{\prime} \in \mathcal{P}$ and $\xi^{\prime}=w^{Q}(\xi):=\left(P^{\prime}, \delta^{\prime}, t^{P^{\prime}}\right)$.

Let $a^{\prime} \in i\left(V_{\delta^{\prime}}\right)=\mathcal{H}\left(W^{P^{\prime}}\right) \otimes V_{\delta^{\prime}}, b^{\prime} \in \mathcal{H}\left(W_{Q^{\prime}}^{P^{\prime}}\right) \otimes V_{\delta^{\prime}} \subset i\left(V_{\delta^{\prime}}\right)$ and let $h \in \mathcal{H}$. We introduce the unitary isomorphism

$$
\sigma:=\psi_{w^{Q}} \otimes \tilde{\delta}_{w^{Q}}: \mathcal{H}\left(W_{Q}^{P}\right) \otimes V_{\delta} \rightarrow \mathcal{H}\left(W_{Q^{\prime}}^{P^{\prime}}\right) \otimes V_{\delta^{\prime}},
$$

and the orthogonal projection

$$
\rho: i\left(V_{\delta}\right) \rightarrow \mathcal{H}\left(W_{Q}^{P}\right) \otimes V_{\delta}
$$

With these notations, put

$$
\begin{aligned}
a & :=\rho\left(\pi\left(w^{Q}, \bar{\xi}^{-1}\right)^{-1}\left(a^{\prime}\right)\right) \in \mathcal{H}\left(W_{Q}^{P}\right) \otimes V_{\delta} \\
b & :=\sigma^{-1}\left(b^{\prime}\right) \in \mathcal{H}\left(W_{Q}^{P}\right) \otimes V_{\delta}
\end{aligned}
$$

We then have, with $c^{Q}(\xi):=\prod_{\alpha \in R_{0,+} \backslash R_{Q,+}} c_{\alpha}(t)$,

$$
f_{a^{\prime}, b^{\prime}}^{w^{Q^{\prime}}}\left(\xi^{\prime}, h\right)=q\left(w^{Q}\right)^{1 / 2} c^{Q}(\xi) f_{Q, a, b}\left(\xi, p_{Q}(h)\right) .
$$

Here $f_{Q, a, b}(\xi, h)=f_{a, b}(\xi, h)$ (with $\left.h \in \mathcal{H}^{Q}, a, b \in \mathcal{H}\left(W_{Q}^{P}\right) \otimes V_{\delta}\right)$ is the matrix coefficient (associated to the pair $a, b$ ) of the representation

$$
\pi^{Q}(\xi):=\operatorname{Ind}_{\mathcal{H}^{P}}^{\mathcal{H}^{Q}} \delta_{t^{P}}
$$

of $\mathcal{H}^{Q}$ (which is tempered and unitary if $\xi \in \Xi_{u}$ ). 
Proof. Choose $r_{P} \in T_{P}$ such that $W_{P} r_{P}$ is the central character of $\delta$, and write $t^{\prime}=w^{Q}(t)$ with $t=r_{P} t^{P}$. Since we are dealing with rational functions of $\xi$ it is sufficient to assume that $\xi$ is regular, i.e. that $t$ is $R_{P}$-regular. We then extend $\pi\left(\xi^{\prime}\right)$ to the completion $\overline{\mathcal{H}}_{t}$ (recall 2.12.3) and study $\pi\left(\xi^{\prime}\right)$ in the light of the isomorphisms (2.18) and (3.5).

We combine, in the decomposition (2.18) applied to the parabolic $P^{\prime}=w^{Q}(P)$ and parameter $t^{\prime}$, the idempotents according to left cosets of $W_{Q^{\prime}}$. In other words, we partition $W_{0} t$ into the sets $w(\Omega)$ with $w \in W^{Q^{\prime}}$ and $\Omega=W_{Q^{\prime}} t^{\prime}=W_{Q^{\prime}}^{P^{\prime}} \varpi^{\prime}$ ( with $\left.\varpi^{\prime}=w^{Q}(\varpi)=W_{P^{\prime}} t^{\prime}\right)$. These sets are evidently unions of the original equivalence classes in formula

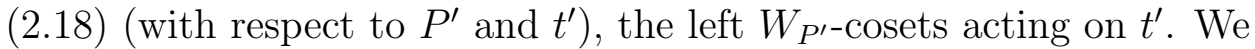
denote the corresponding idempotents by (for all $w \in W^{Q^{\prime}}$ )

$$
e_{w}^{\sharp}:=\sum_{x \in W_{Q^{\prime}}^{w_{Q} Q_{(P)}}} e_{w x \varpi^{\prime}} \cdot
$$

Note that $t^{\prime}$ is $P^{\prime}$-generic, and thus certainly $Q^{\prime}$-generic. The structure formula (2.18) holds therefore, also in terms of the idempotents $e_{w}^{\sharp}$, where we replace in (2.18) the parabolic $P^{\prime}$ by $Q^{\prime}$.

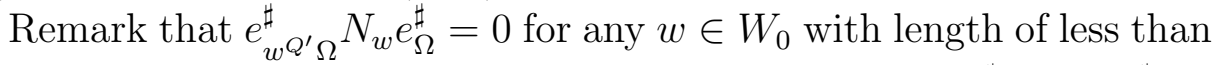
$\left|R_{0,+} \backslash R_{Q}\right|$ (=the length of $w^{Q^{\prime}}$ ). Note by the way that $e_{w Q^{\prime} \Omega}^{\sharp}=e_{W_{Q} t}^{\sharp}$. Thus for all $d \in D^{Q, Q^{\prime}}, d \neq w^{Q^{\prime}}$ we see that $e_{W_{Q} t}^{\sharp} \mathcal{H}^{Q, P}(d) e_{w^{Q} W_{Q} t}^{\sharp}=0$.

Hence for all $h \in \mathcal{H}, a^{\prime} \in i\left(V_{\delta^{\prime}}\right)$ and $b^{\prime} \in \mathcal{H}\left(W_{Q^{\prime}}^{P^{\prime}}\right)$ we have

$$
f_{a^{\prime}, b^{\prime}}^{Q^{\prime}}\left(\xi^{\prime}, h\right)=f_{a^{\prime}, b^{\prime}}\left(\xi^{\prime}, p_{Q}(h) e_{W_{Q} t}^{\sharp} N_{w^{Q^{\prime}}} e_{w^{Q} W_{Q} t}^{\sharp}\right) .
$$

Since $f_{a^{\prime}, b^{\prime}}^{Q^{\prime}}\left(\xi^{\prime}, \mathcal{H} \mathcal{I}_{t}\right)=0$ we can use the analog of formula (4.57) of [14] (we use here that the c-function $c^{Q}(t)$ is $W_{Q}$-invariant, together with the argument in the proof of the Proposition 5.8. This makes that we can evaluate the c-factors at $\left.t^{\prime}\right)$ :

$$
f_{a^{\prime}, b^{\prime}}^{Q^{Q^{\prime}}}\left(\xi^{\prime}, h\right)=q\left(w^{Q}\right)^{1 / 2} c^{Q}(t) f_{a^{\prime}, b^{\prime}}\left(\xi^{\prime}, p_{Q}(h) \iota_{w^{Q^{\prime}}}^{0}\right) .
$$

We use Lemma 5.14 and then rewrite the result using (2.18) and Definition 3.6. Assume that $b^{\prime}=x^{\prime} \otimes v^{\prime}$ and $b=\sigma^{-1}\left(b^{\prime}\right)=x \otimes v$. Then

$$
\begin{aligned}
\iota_{w^{Q^{\prime}}}^{0}\left(b^{\prime}\right) & =\iota_{w^{Q^{\prime}}}^{0}\left(x^{\prime} e_{W_{Q^{\prime}}}^{\sharp} t^{\prime} \otimes v^{\prime}\right) \\
& =e_{W_{Q} t}^{\sharp}\left(\psi_{w^{Q^{\prime}}}\left(x^{\prime}\right) \iota_{w^{Q^{\prime}}}^{0} \otimes v^{\prime}\right) \\
& =e_{W_{Q} t}^{\sharp} \pi\left(w^{Q}, \xi\right)(x \otimes v) \\
& =\pi\left(w^{Q}, \xi\right)(b) .
\end{aligned}
$$


Thus we obtain

$$
\begin{aligned}
f_{a^{\prime}, b^{\prime}}^{w^{Q^{\prime}}}\left(\xi^{\prime}, h\right) & =q\left(w^{Q}\right)^{1 / 2} c^{Q}(\xi) f_{a^{\prime}, \pi\left(w^{Q}, \xi\right)(b)}\left(\xi^{\prime}, p_{Q}(h)\right) \\
& =q\left(w^{Q}\right)^{1 / 2} c^{Q}(\xi) f_{\left(\pi\left(w^{Q}, \bar{\xi}^{-1}\right)^{-1}\left(a^{\prime}\right)\right), b}\left(\xi, p_{Q}(h)\right) \\
& =q\left(w^{Q}\right)^{1 / 2} c^{Q}(\xi) f_{Q, a, b}\left(\xi, p_{Q}(h)\right) .
\end{aligned}
$$

In the second step we use the unitarity of the intertwining operators $\pi\left(w^{Q}, \xi\right)$ to rewrite the matrix coefficient as a coefficient of the induced representation $\pi(\xi)$ (extended holomorphically as in Lemma 5.15 in fact it is a simple special case of this Lemma). Since $b \in \mathcal{H}\left(W_{Q}^{P}\right) \otimes$ $V_{\delta}$ and $p_{Q}(h) \in \mathcal{H}^{Q}$, we can project the vector $\pi\left(w^{Q}, \bar{\xi}^{-1}\right)^{-1}\left(a^{\prime}\right)$ onto $\mathcal{H}\left(W_{Q}^{P}\right) \otimes V_{\delta}$, and consider the result as a matrix coefficient of $\pi^{Q}(\xi)$.

Theorem 5.19. Fix $P \in \mathcal{P}$ and $\delta \in \Delta_{P, W_{P} r_{P}}$. We denote by $\Xi_{P, \delta} \subset \Xi$ the collection of standard induction data of the form $\left(P, \delta, t^{P}\right)$ with $t^{P} \in T^{P}$, and by $\Xi_{P, \delta, u} \subset \Xi_{P, \delta}$ the subset of such triples with $t^{P} \in T_{u}^{P}$. Then for all $d \in D^{Q, P}$ such that $d(P) \subset Q$ and for all $a, b \in i\left(V_{\delta}\right)$, the rational function

$$
\xi \rightarrow c(\xi)^{-1} f_{a, b}^{d}(\xi, h)
$$

is regular in a neighborhood of $\Xi_{P, \delta, u}$.

Proof. We apply Lemma 5.15 with $g=w^{Q} d \in W\left(P, P^{\prime}\right)$ where $P^{\prime}=$ $w^{Q}(d(P)) \subset Q^{\prime}$. Notice that $d^{\prime}=w^{Q^{\prime}}$. Put $\xi^{\prime}=g(\xi)$ and

$$
\begin{aligned}
& a^{\prime}=\pi\left(g, \bar{\xi}^{-1}\right)(a) \\
& \tilde{b}^{\prime}=\pi(g, \xi)(b) .
\end{aligned}
$$

We obtain

$$
c(\xi)^{-1} f_{a, b}^{d}(\xi, h)=c(\xi)^{-1} f_{a^{\prime}, \tilde{b}^{\prime}}^{Q^{\prime}}\left(\xi^{\prime}, h\right) .
$$

Now we can uniquely decompose $\tilde{b}^{\prime}$ in the following way

$$
\tilde{b}^{\prime}=\pi\left(\xi^{\prime}, \tilde{h}\right)\left(b^{\prime}\right)
$$

with $\tilde{h} \in \mathcal{H}\left(W^{Q^{\prime}}\right)$ and $b^{\prime} \in \mathcal{H}\left(W_{Q^{\prime}}^{P^{\prime}}\right) \otimes V_{\delta^{\prime}} \subset i\left(V_{\delta^{\prime}}\right)$. With the help of Lemma 5.14 we get

$$
c(\xi)^{-1} f_{a, b}^{d}(\xi, h)=c(\xi)^{-1} f_{a^{\prime}, b^{\prime}}^{w^{Q^{\prime}}}\left(\xi^{\prime}, h \tilde{h}\right) .
$$

We can now apply Theorem 5.18 with respect to $P_{0}:=d(P) \subset Q$. We put $\xi_{0}:=d(\xi)$ and

$$
\begin{aligned}
& a_{0}:=\rho\left(\pi\left(w^{Q}, \bar{\xi}_{0}^{-1}\right)^{-1}\left(a^{\prime}\right)\right) \in \mathcal{H}\left(W_{Q}^{P_{0}}\right) \otimes V_{\delta} \\
& b_{0}:=\sigma^{-1}\left(b^{\prime}\right) \in \mathcal{H}\left(W_{Q}^{P_{0}}\right) \otimes V_{\delta}
\end{aligned}
$$


to obtain:

$$
c(\xi)^{-1} f_{a, b}^{d}(\xi, h)=q\left(w^{Q}\right)^{1 / 2}\left(c(\xi)^{-1} c\left(\xi_{0}\right)\right) c_{Q}\left(\xi_{0}\right)^{-1} f_{Q, a_{0}, b_{0}}\left(\xi_{0}, p_{Q}(h \tilde{h})\right),
$$

where in general for $Q \supset P$ we denote

$$
c_{Q}(\xi):=\prod_{\alpha \in R_{Q,+} \backslash R_{P,+}} c_{\alpha}(t) .
$$

The regularity of the normalization factor $c_{Q}\left(\xi_{0}\right)^{-1}$ as a function of $\xi_{0}$ (and thus as a function of $\left.\xi=d^{-1}\left(\xi_{0}\right)\right)$ follows from 14, Theorem 3.25, when we consider the tempered residual coset $r_{P} T_{u}^{P} \subset T$ for the Hecke algebra $\mathcal{H}^{Q}$ (instead of $\mathcal{H}$ itself). It is a simple special case of Proposition 8.8(v). Similarly, the regularity of $c(\xi)^{-1} c\left(\xi_{0}\right)$ is asserted by Proposition [8.8(iv). By the regularity of the various intertwining operators we have used (cf. Theorem 3.3) it is clear that also $a_{0}, b_{0}$ are also rational and regular on $\Xi_{P, \delta, u}$. We have finished the proof.

From the proof of Theorem 5.19 we obtain

Corollary 5.20. Thus, the final conclusion of all these considerations is that for all $h \in \mathcal{H}, P \in \mathcal{P}, \delta \in \Delta_{P}$, and $a, b \in i\left(V_{\delta}\right)$ fixed, the function

$$
\Xi_{P, \delta, u} \times \mathcal{H}^{Q} \ni\left(\xi, h^{Q}\right) \rightarrow c(\xi)^{-1} f_{a, b}^{Q}\left(\xi, h^{Q} h\right)
$$

is a linear combination with coefficient functions which are regular rational functions on $\Xi_{P, \delta, u}$, of normalized matrix coefficients

$$
c_{Q}(d(\xi))^{-1} f_{Q, a^{\prime}, b^{\prime}}\left(d(\xi), h^{Q}\right)
$$

of induced representations of $\mathcal{H}^{Q}$ of the form $\pi^{Q}(d(\xi)$ ) (where $d$ ranges over the Weyl group elements $d \in D^{Q, P}$ such that $\left.d(P) \subset Q\right)$.

\section{Proof of the main theorem}

\subsection{Uniform estimates for the coefficients of $\pi(\xi)$}

Recall that $X^{+}$is the cone $\left\{x \in X \mid\left\langle x, \alpha^{\vee}\right\rangle \geq 0\right.$ for all $\left.\alpha \in R_{1,+}\right\}$. We put $Z_{X}=X^{+} \cap X^{-}$. This is a sublattice of elements in $X$ with length 0 . Recall that $Q$ denotes the root lattice. The sublattice $Q \oplus$ $Z_{X} \subset X$ has finite index in $X$. If $x=x_{Q}+x_{Z} \in Q^{+}+Z_{X}$ then

$$
\mathcal{N}(x)=x_{Q}\left(2 \rho^{\vee}\right)+\left\|x_{Z}\right\| .
$$

Let us show that $Q^{+}$is finitely generated over $\mathbb{Z}_{+}$. For each fundamental weight $\delta_{i}$, let $q_{i}=m_{i} \delta_{i}$ be the least multiple of $\delta_{i}$ such that $q_{i} \in Q$ (thus $m_{i} \in \mathbb{N}=\{1,2,3, \cdots\}$ is a divisor of the index $[P: Q]$ ). These 
multiples generate over $\mathbb{Z}_{+}$a cone $C^{+} \subset Q^{+}$. Let $F$ be the fundamental domain $F=\left\{\sum_{i} t_{i} q_{i} \mid t_{i} \in[0,1)\right\}$ of $C$, and let $F_{Q}=F \cap Q \subset Q^{+}$ (a finite set). Clearly $F_{Q}$ and the $\left\{q_{i}\right\}$ generate $Q^{+}$over $\mathbb{Z}_{+}$. Let $x_{1}, \cdots, x_{m}, x_{m+1}, \cdots, x_{N} \in X^{+}$such that $x_{1}, \cdots, x_{m}$ is a set of $\mathbb{Z}_{+^{-}}$ generators of $Q^{+}$and that $x_{m+1}, \cdots, x_{N} \in Z_{X}$ is a $\mathbb{Z}$-basis of $Z_{X}$. By (6.1) we see that there exists a constant $K>0$ such that for all $x \in Q^{+}+X_{Z}$ and all decompositions $x=\sum l_{i} x_{i}$ with $l_{i} \geq 0$ if $i \leq m$, we have

$$
\sum\left|l_{i}\right| \leq K \mathcal{N}(x)
$$

(just observe that $x_{i}\left(2 \rho^{\vee}\right) \geq 1$ if $\left.i \in 1 \ldots, m\right)$. We fix such a $K>0$.

We define a function $\nu$ on $T_{\text {rs }}$ by

$$
\begin{aligned}
\nu(t)=\max \left(\left\{\left|x_{i}(t)\right|\right.\right. & \mid i=m+1, \ldots, N\} \\
& \left.\cup\left\{\left|x_{i}(w t)\right| \mid i=1, \ldots, m ; w \in W_{0}\right\}\right) .
\end{aligned}
$$

The positive real cone spanned by the elements $w x_{i}\left(w \in W_{0}, i=\right.$ $1, \ldots, m)$ and $\pm x_{i}(i=m+1, \ldots, N)$ is the full dual of $\operatorname{Lie}\left(T_{r s}\right)$. Therefore the function $\log (\nu) \circ \exp$ is a norm on $\operatorname{Lie}\left(T_{r s}\right)$.

Theorem 6.1. Let $R>1, P \in \mathcal{P}$, and $\delta \in \Delta_{P}$ be given. Choose a set $x_{i} \in X^{+}$as above and let $K$ and $\nu$ be as above. We use the notation $\nu(|\xi|):=\nu\left(\left|t^{P}\right|\right)$ for $\xi=\left(P, \delta, t^{P}\right) \in \Xi_{P, \delta}$. Define a compact neighborhood $D^{P}(R) \subset \Xi_{P, \delta}$ of $\Xi_{P, \delta, u} \subset \Xi_{P, \delta}$ by $D^{P}(R)=\left\{\xi \in \Xi_{P, \delta} \mid\right.$ $\nu(|\xi|) \leq R\}$.

There exists a $d \in \mathbb{N}$, and there exists a constant $c>0$ (depending on $R$ only) such that for all $w \in W$, for all $a, b \in i\left(V_{\delta}\right)$, and for all $\xi \in D^{P}(R)$, the matrix coefficient $f_{a, b}\left(\xi, N_{w}\right)$ satisfies

$$
\left|f_{a, b}\left(\xi, N_{w}\right)\right| \leq c\|a\|\|b\|(1+\mathcal{N}(w))^{d} \nu(|\xi|)^{K \mathcal{N}(w)}
$$

Proof. Using 14, equation (2.27) (also see the proof of Lemma 6.11) we see that it is equivalent to show that $f_{a, b}\left(\xi, N_{u} \theta_{x} N_{v}\right)$ can be estimated by the right hand side of (6.4) with $w=x$, for all $u, v \in W_{0}$ and $x \in X^{+}$. By applying right (resp. left) translations of the matrix coefficient $f_{a, b}(\xi)$ by $N_{v}$ (resp. $N_{u}$ ) and by a set of representatives of the finite quotient $X /\left(Q+Z_{X}\right)$ we see that we may further reduce to proving the estimates (6.4) for $w=x \in Q^{+}+Z_{X}$.

Recall (cf. 14, Proposition 4.20 and its proof) that the the eigenvalues of the matrix of $\pi\left(\xi, \theta_{x}\right)$ are of the form $x\left(w_{i}\left(r_{j} t^{P}\right)\right)$. Here the $r_{j} \in T_{P}$ are the generalized $X_{P}$-eigenvalues of the discrete series representation $\delta$. By Casselman's criterion we know therefore that for all $x \in X^{+}, x\left(w_{i}\left(r_{j}\right)\right) \leq 1$. This implies that for all $i \in 1, \ldots, m$, and for all $\xi \in \Xi_{P, \delta}$, the eigenvalues of $\pi\left(\xi, \theta_{x_{i}}\right)$ are bounded by $\nu(|\xi|)$. 
Then Lemma 7.1 allows to estimate the norm of $\pi\left(\xi, \theta_{l_{i} x_{i}}\right)$, by dividing $\pi\left(\xi, \theta_{x_{i}}\right)$ by $\nu(|\xi|)$.

Taking into account the fact that $D^{R}(P)$ is compact, one sees that the norm of $\pi\left(\xi, \theta_{x_{i}}\right)$ is bounded if $\xi$ is in $D^{R}(P)$. By a simple product formula, one estimates the norm of $\pi\left(\xi, \theta_{x}\right)$. These estimates together with equation (6.2) imply the desired result.

Corollary 6.2. For all constant coefficient differential operators $D$ on $\Xi_{P, \delta}$ there exist constants $d \in \mathbb{N}$ and $c>0$ such that for all $\xi \in \Xi_{P, \delta, u}$, for all $a, b \in i\left(V_{\delta}\right)$, and for all $w \in W$

$$
\left|D f_{a, b}\left(\xi, N_{w}\right)\right| \leq c\|a\|\|b\|(1+\mathcal{N}(w))^{d} .
$$

Proof. This is a standard application of the Cauchy integral formula, starting with equation (6.4). Choose a basis $x_{1}, \ldots, x_{p}$ of the character lattice $X^{P}$ of $T^{P}$, and let $y_{1}, \ldots, y_{p}$ be the dual basis. Let $C_{\epsilon}:=\{v \in$ $\left.\operatorname{Lie}\left(T^{P}\right)|\forall i:| x_{i}(v) \mid=\epsilon\right\}$. We may assume that $D$ is of the form $D=D^{\alpha}:=y_{1}^{\alpha_{1}} \ldots y_{p}^{\alpha_{p}}$. By the holomorphicity of $f_{a, b}$ we have, for a suitable constant $C_{\alpha}>0$ and any choice of a sequence of radii $\epsilon(w)$ :

$$
D^{\alpha} f_{a, b}\left(\xi, N_{w}\right)=C_{\alpha} \int_{v \in C_{\epsilon(w)}} \frac{f_{a, b}\left(\exp (v) \cdot \xi, N_{w}\right)}{\prod_{i} x_{i}(v)^{\alpha_{i}+1}} d x_{1} \wedge \cdots \wedge d x_{p}
$$

Now use the estimates of Theorem 6.1 with the sequence $\epsilon(w)$ chosen such that $r(w):=\max \left\{\nu(|\exp (v)|) \mid v \in C_{\epsilon(w)}\right\}$ is equal to

$$
1+1 /(1+\mathcal{N}(w)) \text {. }
$$

But $\log (\nu) \circ \exp$ is a norm on $\operatorname{Lie}\left(T^{P}\right)$, as well as $\operatorname{Sup}_{i}\left|x_{i}(v)\right|$. They are equivalent. Moreover $\log (1+x) \geq k^{\prime} x$ for $x \in[0,1]$, for some $k^{\prime}>0$. Together with 6.7 this implies that there exists a constant $k>0$ such that $\epsilon(w) \geq k /(1+\mathcal{N}(w))$. So equation (6.6) yields the estimate (for some constant $\left.c^{\prime}>0\right)$

$$
\left|D^{\alpha} f_{a, b}\left(\xi, N_{w}\right)\right| \leq c^{\prime}\|a\|\|b\|(1+\mathcal{N}(w))^{d+|\alpha|}(1+1 /(1+\mathcal{N}(w)))^{K \mathcal{N}(w)} .
$$

This easily leads to the desired result.

Corollary 6.3. We have $\mathcal{F}(\mathcal{S}) \subset C^{\infty}\left(\Xi_{u}, \operatorname{End}\left(\mathcal{V}_{\Xi}\right)\right)^{\mathcal{W}}$. The restriction $\mathcal{F}_{\mathcal{S}}$ of $\mathcal{F}$ to $\mathcal{S}$ defines a continuous map $\mathcal{F}_{\mathcal{S}}: \mathcal{S} \rightarrow C^{\infty}\left(\Xi_{u}, \operatorname{End}\left(\mathcal{V}_{\Xi}\right)\right)^{\mathcal{W}}$.

Proof. The equivariance of the sections in the image is clear. Recall that $\mathcal{F}\left(N_{w}\right) \in \operatorname{Pol}\left(\Xi_{u}, \operatorname{End}\left(\mathcal{V}_{\Xi}\right)\right)$ is defined by $\mathcal{F}\left(N_{w}\right)(\xi)=\pi\left(\xi, N_{w}\right)$.

Hence by the estimates of Corollary 6.2 we see that for any continuous seminorm $p$ on $C^{\infty}\left(\Xi_{u}, \operatorname{End}\left(\mathcal{V}_{\Xi}\right)\right)$ there exist constants $C>0$ and $d \in \mathbb{Z}_{+}$such that $p\left(\mathcal{F}\left(N_{w}\right)\right) \leq C(1+\mathcal{N}(w))^{d}$. 
Let $b \in \mathbb{Z}_{+}$be such that $0<C_{b}:=\sum_{w \in W}(1+\mathcal{N}(w))^{-b}<\infty$, and let $q=q_{p}$ denote the continuous seminorm on $\mathcal{S}$ defined by $q(x):=$ $C C_{b} \sup _{w}\left|\left(x, N_{w}\right)\right|(1+\mathcal{N}(w))^{d+b}$. Then $p\left(\mathcal{F}_{\mathcal{S}}(x)\right) \leq q(x)$ for all $x \in \mathcal{H}$, implying that $\mathcal{F}_{\mathcal{S}}$ is a continuous map as claimed.

\subsection{Uniform estimates of the difference of a coefficient and its constant term}

We now introduce the important notion of a normalized smooth family of coefficients:

Definition 6.4. Let $P \in \mathcal{P}$ and let $\delta \in \Delta_{P}$ be an irreducible discrete series of $\mathcal{H}_{P}$ with central character $W_{P} r_{P} \in W_{P} \backslash T_{P}$. We put $\xi=$ $\left(P, \delta, t^{P}\right) \in \Xi_{P, \delta, u}$. A smooth family of coefficients of $\pi(\xi), \xi \in \Xi_{P, \delta, u}$ is a family of linear functionals on $\mathcal{H}$ of the form:

$$
\mathcal{H} \ni h \mapsto \operatorname{Tr}(\sigma(\xi) \pi(\xi)(h)),
$$

where $\sigma$ is a section of $C^{\infty}\left(\Xi_{P, \delta, u}, \operatorname{End}\left(\mathcal{V}_{\Xi}\right)\right)$.

A smooth section $\sigma \in C^{\infty}\left(\Xi_{P, \delta, u}, \operatorname{End}\left(\mathcal{V}_{\Xi}\right)\right)$ is called normalized smooth when it is divisible (in the $C^{\infty}\left(\Xi_{P, \delta, u}\right)$-module $C^{\infty}\left(\Xi_{P, \delta, u}\right.$, End $\left.\left(\mathcal{V}_{\Xi}\right)\right)$ ) by the smooth function $\left\{\xi \rightarrow c^{-1}(\xi)\right\} \in C^{\infty}\left(\Xi_{P, \delta, u}\right)$ (cf. Proposition [8.8).

A normalized smooth family of coefficients of $\pi(\xi), \xi \in \Xi_{P, \delta, u}$ is a smooth family of coefficients (6.9) for which $\sigma$ is normalized smooth.

Remark 6.5. We frequently use $t^{P}$ rather than $\xi=\left(P, \delta, t^{P}\right)$ as the parameter of a family of coefficients.

Corollary 6.6. It follows directly from the definitions that smooth (resp. normalized smooth) families of coefficients of $\pi(\xi), \xi \in \Xi_{P, \delta, u}$, are stable under left and right translations by elements of $h \in \mathcal{H}$.

Moreover, Corollary 5.20 implies that:

Corollary 6.7. The restriction to $\mathcal{H}^{Q}$ of the constant term of a normalized smooth family of $\pi(\xi)=\pi\left(P, \delta, t^{P}\right), t^{P} \in T_{u}^{P}$, along $Q \in \mathcal{P}$ is a finite sum of terms, each of these being a normalized smooth family of coefficients of $\pi^{Q}(d(\xi))$, where $d$ is some Weyl group element with $d(P) \subset Q$.

Lemma 6.8. Assume $Z_{X}=\{0\}$. Let $\Xi_{P, \delta, t^{P}} \ni \xi \rightarrow \Phi_{\xi}$ be a smooth family of coefficients.

Let $\alpha \in F_{0}$, and put $Q=F_{0} \backslash\{\alpha\}$.

Let $\|\cdot\|$ denote a norm which comes from a $W_{0}$-invariant euclidean structure on $X \otimes_{\mathbb{Z}} \mathbb{R}$. 
Let $a>0$ and let $X_{a}^{+}$denote the cone (over $\mathbb{Z}_{+}$) $X_{a}^{+}=\left\{x \in X^{+} \mid\right.$ $\left.\left\langle x, \alpha^{\vee}\right\rangle>a\|x\|\right\}$. Then there exists $C, b>0$ such that

$$
\left|\left(\Phi_{\xi}-\Phi_{\xi}^{Q}\right)\left(N_{u} \theta_{x} N_{v}\right)\right| \leq C e^{-b\|x\|},
$$

for all $x \in X_{a}^{+}, \xi \in \Xi_{P, \delta, u}, u \in W_{Q}$, and $v \in W_{0}$.

Proof. Recall that the lattice $X$ contains the root lattice $Q\left(R_{0}\right)$, and hence an integral multiple of the weight lattice $P\left(R_{0}\right), k P\left(R_{0}\right)$ say. We put $X^{\prime}=k P\left(R_{0}\right) \subset X$ and we identify $X^{\prime}$ with $\mathbb{Z}^{l}$ via a basis of $X^{\prime}$ consisting of the elements $\left(k \delta_{\beta}\right), \beta \in F_{0}$ (where the $\delta_{\beta}$ are the fundamental weights), ordered in such a way that $e_{1}=k \delta_{\alpha}$. The temperedness of $\pi\left(P, \delta, t^{P}\right), t^{P} \in T_{u}^{P}$, and the fact that its central character is given by $t=r_{P} t^{P}$ imply that the possible eigenvalues of $\pi\left(P, \delta, t^{P}\right)\left(\theta_{k \delta_{\beta}}\right)$ are among the $w t\left(k \delta_{\beta}\right)$ with $w \in W_{0}$ such that $\left|w t\left(k \delta_{\beta}\right)\right| \leq 1$. Moreover the modulus of $w t\left(k \delta_{\beta}\right)$, hence of $w t\left(e_{1}\right)$, does not depend on $t^{P} \in T_{u}^{P}$.

Hence if $u=v=e$ and $x \in X^{\prime}=k P\left(R_{0}\right)$, (6.10) follows, in view of the definition of the constant term, from Lemma 7.3 .

Let us now derive the general case of (6.10) from this special case. Since $Z_{X}=\{0\}, X^{\prime}$ is of finite index in $X$. One can assume that $a$ is small enough, in such a way that $X_{a}^{+}$is nonempty, otherwise there is nothing to prove. Let $x \in X_{a}^{+}$and let $y$ be the orthogonal projection of $x$ on the line $\mathbb{R} \delta_{\alpha}$. By definition of $X_{Q}$ we have $x-y \in X_{Q}$, and since $\left\langle x-y, \beta^{\vee}\right\rangle=\left\langle x, \beta^{\vee}\right\rangle$ for all $\beta \in Q$ we find that in fact $x-y \in X_{Q}^{+}$. Thus $x-y$ is a nonnegative linear combination of the fundamental weights $\delta_{\beta}^{Q}$ of $R_{Q}$. It is a basic fact that the fundamental weights of a root system (with given basis of simple roots) have nonnegative rational coefficients in the basis of its simple roots (indeed, this statement reduces to the case of an irreducible root system, in which case the indecomposability of the Cartan matrix implies that these coefficients are in fact strictly positive). Hence we have $x-y \in X_{Q}^{+} \subset \mathbb{Q}_{+} Q$. Since $\left\langle\beta, \alpha^{\vee}\right\rangle \leq 0$ for all $\beta \in Q$, we see that $\left\langle y, \alpha^{\vee}\right\rangle \geq\left\langle x, \alpha^{\vee}\right\rangle>a\|x\| \geq a\|y\|$. Hence $k \delta_{\alpha} \in X_{a}^{+}$.

Let $\left(x_{1}, \ldots, x_{r}\right)$ be a set of representatives in $X$ of $X / X^{\prime}$. Let us show that one can choose the $x_{i}$ in $-X_{a}^{+}$. Our claim is a consequence of the following fact. If $y \in X$, one has $y+n \delta_{\alpha} \in X_{a}^{+}$for $n$ large. In fact by the triangular inequality one has:

$$
\left\langle y+n \delta_{\alpha}, \alpha^{\vee}\right\rangle-a\left\|y+n \delta_{\alpha}\right\| \geq n\left(\left\langle\delta_{\alpha}, \alpha^{\vee}\right\rangle-a\left\|\delta_{\alpha}\right\|\right)+\alpha(y)-a\|y\|
$$

Thus, if $x \in X_{a}^{+}$and $x=x^{\prime}+x_{i}$, for some $x^{\prime} \in X^{\prime}$ and some $i$, one has $x^{\prime} \in X_{a}^{\prime+}$. To get the estimates, one applies the previous estimates to the translates of the family $\Phi_{\xi}$ by the $N_{u}$ (from the left), and by $\theta_{x_{i}} N_{v}$ 
(from the right), which are smooth families of coefficients themselves (cf. Corollary 6.6), and taking into account Corollary 5.4.

\subsection{Wave packets}

Recall that $\mathcal{J}$ was introduced as the adjoint of $\mathcal{F}$. Thus if $\sigma \in$ $L_{2}\left(\Xi_{P, \delta, u}, \operatorname{End}\left(\mathcal{V}_{\Xi}\right), \mu_{P l}\right)$ then $\mathcal{J}(\sigma) \in L_{2}(\mathcal{H})$, and is completely characterized by the value of $(\mathcal{J}(\sigma), h)$ where $h \in \mathcal{H}$. We have, using Theorem 3.6. that

$$
\begin{aligned}
\mathcal{J}(\sigma)(h): & =\left(\mathcal{J}(\sigma)^{*}, h\right)=\left(h^{*}, \mathcal{J}(\sigma)\right)= \\
& =\left(\mathcal{F}\left(h^{*}\right), \sigma\right)=\mu_{\mathcal{R}, \delta} \int_{\Xi_{P, \delta, u}} \operatorname{Tr}(\sigma(\xi) \pi(\xi, h))|c(\xi)|^{-2} d \xi,
\end{aligned}
$$

where $\mu_{\mathcal{R}, \delta}=q\left(w^{P}\right)^{-1}\left|\mathcal{W}_{P} / \mathcal{K}_{P}\right|^{-1} \mu_{\mathcal{R}_{P}, P l}(\{\delta\})>0$.

Recall Definition 4.1. Assume that $\tilde{\sigma}=c\left(w^{P} \cdot\right) \sigma \in \mathcal{C}\left(\Xi_{u}, \operatorname{End}\left(\mathcal{V}_{\Xi}\right)\right)$ (in other words, $\sigma \in C^{\infty}\left(\Xi_{P, \delta, u}, \operatorname{End}\left(\mathcal{V}_{\Xi}\right)\right)$ ).

Denote by $\Phi^{\sigma}$ the smooth family of coefficients $\Phi_{\xi}^{\sigma}(h)=\operatorname{Tr}(\sigma(\xi) \pi(\xi, h))$ associated with $\sigma$. Then we have by (6.11) (with $h \in \mathcal{H}$ )

$$
\mathcal{J}(\tilde{\sigma})(h)=\mu_{\mathcal{R}, \delta} W_{\sigma}(h),
$$

where we put for any $\sigma \in C^{\infty}\left(\Xi_{P, \delta, u}, \operatorname{End}\left(\mathcal{V}_{\Xi}\right)\right)$,

$$
\begin{aligned}
W_{\sigma}(h): & =\int_{\Xi_{P, \delta, u}} \Phi_{\xi}^{\sigma}(h) c^{-1}(\xi) d \xi \\
& =\int_{\Xi_{P, \delta, u}} \operatorname{Tr}(\sigma(\xi) \pi(\xi, h)) c^{-1}(\xi) d \xi
\end{aligned}
$$

Theorem 6.9. For every $k \in \mathbb{Z}_{+}$, there exists a continuous seminorm $p_{k}$ on $C^{\infty}\left(\Xi_{P, \delta, u}, \operatorname{End}\left(\mathcal{V}_{\Xi}\right)\right)$ such that

$$
\left|W_{\sigma}\left(N_{u} \theta_{x} N_{v}\right)\right| \leq(1+\|x\|)^{-k} p_{k}(\sigma),
$$

for all $x \in X^{+}, u, v \in W_{0}$ and $\sigma \in C^{\infty}\left(\Xi_{P, \delta, u}, \operatorname{End}\left(\mathcal{V}_{\Xi}\right)\right)$.

Proof. First, by using right and left translations by the $N_{w}, w \in W_{0}$, and Corollary 6.6, it is enough to prove (6.14) for $u=v=1$. Thus, we assume $u=v=1$ in the following.

The proof is by induction on the rank of $X$. The statement is clear if the rank of $X$ is zero. One assumes the theorem is true for lattices of rank strictly smaller than rank of $X$.

For the induction step we consider two cases, namely the case where $Z_{X} \neq 0$ (first case), and the case where $Z_{X}=0$ (second case). 
First case. In this case the semisimple quotient $\mathcal{H}_{F_{0}}$ of $\mathcal{H}=\mathcal{H}^{F_{0}}$ has smaller rank than $\mathcal{H}$. Recall the results of Proposition 2.2 and Proposition 2.3. Let us denote the semisimple quotient $\mathcal{H}_{F_{0}}$ of $\mathcal{H}$ by $\mathcal{H}_{0}$, its root datum $\mathcal{R}_{F_{0}}$ by $\mathcal{R}_{0}$ etc.

We have $T_{P} \subset T_{0}$ and $T^{P} \supset T^{0}$. Let $T_{0}^{P}=\left(T_{0}\right)^{P}$ be the connected component of $e$ of the intersection $T_{0} \cap T^{P}$. Then the product map $T_{P} \times T_{0}^{P} \rightarrow T_{0}$ is a finite covering, as is the product map $T_{0}^{P} \times T^{0} \rightarrow T^{P}$. Let $\xi=\left(P, \delta, t^{P}\right)$ and suppose that $t^{P}=t_{0}^{P} t^{0}$ for $t_{0}^{P} \in T_{0, u}^{P}$ and $t^{0} \in T_{u}^{0}$. Let $\xi_{0}=\left(P, \delta, t_{0}^{P}\right) \in \Xi_{\mathcal{R}_{0}, P, \delta, u}$ denote the standard induction datum for $\mathcal{H}_{0}$. Recall the epimorphism $\phi_{t^{0}}: \mathcal{H} \rightarrow \mathcal{H}_{0}$ of Proposition 2.3. It is easy to see that $\pi(\xi)=\pi\left(\xi_{0}\right)_{t^{0}}$, the pull back of the representation $\pi\left(\xi_{0}\right)$ of $\mathcal{H}_{0}$ to $\mathcal{H}$ via $\phi_{t^{0}}$. This implies that

$$
\pi(\xi)\left(\theta_{x}\right)=t^{0}(x) \pi\left(\xi_{0}\right)\left(\theta_{x_{0}}\right)
$$

for all $x \in X$, where $x_{0} \in X_{0}$ is the canonical image of $x$ in $X_{0}$.

Hence, since $c(\xi)=c\left(\xi_{0}\right)$ (indeed, use Definition 8.7 and observe that $\alpha(t)=\alpha\left(r_{P} t^{P}\right)=\alpha\left(r_{P} t_{0}^{P} t^{0}\right)=\alpha\left(r_{P} t_{0}^{P}\right)$ for all $\left.\alpha \in R_{0}\right)$ and since

$$
\int_{T_{u}^{P}} f\left(t^{P}\right) d t^{P}=\int_{T_{0, u}^{P} \times T_{u}^{0}} f\left(t_{0}^{P} t^{0}\right) d t_{0}^{P} d t^{0}
$$

for all integrable functions $f$ on $T_{u}^{P}$, we have

$$
W_{\sigma}\left(\theta_{x}\right)=W_{0, \sigma_{x}}\left(\theta_{x_{0}}\right) \text {, }
$$

where $\sigma_{x} \in C^{\infty}\left(\Xi_{\mathcal{R}_{0}, P, \delta, u}, \operatorname{End}\left(\mathcal{V}_{\Xi_{0}}\right)\right)$ is defined by

$$
\begin{aligned}
\sigma_{x}\left(\xi_{0}\right) & =\int_{T_{u}^{0}} t^{0}(x) \sigma\left(t_{0}^{P} t^{0}\right) d t^{0} \\
& =\int_{T_{u}^{0}} t^{0}\left(x^{0}\right) \sigma\left(t_{0}^{P} t^{0}\right) d t^{0},
\end{aligned}
$$

and where $x^{0}$ is the canonical image of $x$ in $X^{0}$.

From equation (6.18) it is clear, by harmonic analysis on the torus $T_{0, u}^{P} \times T_{u}^{0}$, that for all $k \in \mathbb{Z}_{+}$and all continuous seminorms $q$ on $C^{\infty}\left(\Xi_{\mathcal{R}_{0}, P, \delta, u}\right.$, End $\left.\left(\mathcal{V}_{\Xi_{0}}\right)\right)$, there exists a continuous seminorm $p=p_{q, k}$ on $C^{\infty}\left(\Xi_{P, \delta, u}\right.$, End $\left.\left(\mathcal{V}_{\Xi}\right)\right)$ such that

$$
q\left(\sigma_{x}\right)=\left(1+\left\|x^{0}\right\|\right)^{-k} p(\sigma)
$$

for all $x \in X$ and for all $\sigma \in C^{\infty}\left(\Xi_{P, \delta, u}, \operatorname{End}\left(\mathcal{V}_{\Xi}\right)\right)$.

Now apply the induction hypothesis to $W_{0, \sigma_{x}}$. In view of (6.17) and (6.19) this yields the induction step in the first case.

Second case. We now consider the case $Z_{X}=0$. If $\alpha \in F_{0}$ and $a>0$, one defines $X_{\alpha, a}^{+}=\left\{x \in X^{+} \mid\left\langle x, \alpha^{\vee}\right\rangle>a\|x\|\right\}$. We first prove that:

$$
\cup_{\alpha \in F_{0}} X_{\alpha, a}^{+}=X^{+} \backslash\{0\} \text {, for all } a \text { small enough }
$$


Let $x \in X^{+} \backslash\{0\}$. We write

$$
x=\sum_{\alpha \in F_{0}}\left\langle x, \alpha^{\vee}\right\rangle \delta_{\alpha}
$$

where $\left(\delta_{\alpha}\right)$ are the fundamental weights. For $a>0$ small enough, one has, by equivalence of norms in finite dimensional vector spaces:

$$
2 a\|x\| \leq \sup _{\alpha \in F_{0}}\left|\left\langle x, \alpha^{\vee}\right\rangle\right|, x \in X^{+}
$$

Then, for $x \in X^{+} \backslash\{0\}$, choose $\alpha \in F_{0}$ with $\left\langle x, \alpha^{\vee}\right\rangle$ maximal. From (6.21), one has $\left\langle x, \alpha^{\vee}\right\rangle \geq 2 a\|x\|$, hence, as $\|x\| \neq 0$ :

$$
\left\langle x, \alpha^{\vee}\right\rangle>a\|x\| \text {, i.e. } x \in X_{\alpha, a}^{+}
$$

which proves (6.20).

Hence it is enough to prove the estimates for $x \in X_{\alpha, a}^{+}$. Let $Q=$ $F_{0} \backslash\{\alpha\}$. Then it follows from Lemma 6.8, that for some $b>0$, and $C>0$, one has:

$$
\left|\Phi_{t^{P}}^{\sigma}\left(\theta_{x}\right)-\Phi_{t^{P}}^{\sigma, Q}\left(\theta_{x}\right)\right| \leq C e^{-b\|x\|}, \quad \text { for all } x \in X_{\alpha, a}^{+}, \quad t^{P} \in T_{u}^{P}
$$

By integration of this inequality over $T_{u}^{P}$ against the continuous function $\left|c^{-1}(\xi)\right|$ it suffices to prove the estimates (6.14) after replacing $\Phi_{t^{P}}^{\sigma}$ by $\Phi_{t^{P}}^{\sigma, Q}$. But by Corollary [5.20, the restriction to $\mathcal{H}^{Q}$ of the constant term $\Phi_{t^{P}}^{\sigma, Q} c^{-1}(\xi)$ of the normalized smooth family $\Phi_{t^{P}}^{\sigma} c^{-1}(\xi)$ of coefficients is a sum of normalized smooth families of coefficients for $\mathcal{R}_{Q}=\left(X, Y, R_{Q}, R_{Q}^{\vee}, Q\right)$. This brings us back at the first case of the induction step, thus finishing the proof.

Corollary 6.10. It follows from Theorem [6.9, (6.12) and Lemma 6.11 that $\mathcal{J}(\sigma) \in \mathcal{S}$ for all $\sigma \in \mathcal{C}\left(\Xi_{P, \delta, u}\right.$, End $\left.\left(\mathcal{V}_{\Xi}\right)\right)$, and that $\mathcal{J}_{\mathcal{C}}$ : $\mathcal{C}\left(\Xi_{P, \delta, u}, \operatorname{End}\left(\mathcal{V}_{\Xi}\right)\right) \rightarrow \mathcal{S}$ is contiunous.

By Lemma 4.2 we see that in particular $\mathcal{J}(\sigma) \in \mathcal{S}$ for all $\sigma \in$ $C^{\infty}\left(\Xi_{P, \delta, u}, \operatorname{End}\left(\mathcal{V}_{\Xi}\right)\right)$.

\subsection{End of the proof of the main Theorem}

We start with a basic technical lemma:

Lemma 6.11. Let $n \in \mathbb{Z}$. There exists a constant $C_{n}$ with the following property. For all $f \in \mathcal{H}^{*}$ for which there exists $C>0$ such that:

$$
\left|f\left(T_{u} \theta_{x} T_{v}\right)\right| \leq C(1+\|x\|)^{-n}, u, v \in W_{0}, x \in X^{+}
$$

then one has:

$$
\left|f\left(N_{w}\right)\right| \leq C_{n} C(1+\mathcal{N}(w))^{-n}, \quad w \in W
$$


Proof. As in [14] (2.25), one writes, for $w=u x v$, with $u, v \in W_{0}, x \in$ $X^{+}$

$$
N_{w}=\sum_{u, v \in W_{0}} c_{w,\left(u^{\prime}, v^{\prime}\right)} T_{u^{\prime}} \theta_{x} T_{v^{\prime}}
$$

where the real coefficients $c_{u x v,\left(u^{\prime}, v^{\prime}\right)}$ and $c_{u y v,\left(u^{\prime}, v^{\prime}\right)}$ are equal if $x$ and $y$ belong to the same facets of the cone $X^{+}$. The number of facets being finite, one sees, by using the assumption (6.22), that there exists $C^{\prime}$ such that:

$$
\mid f\left(N_{u x v} \mid \leq C^{\prime} C(1+\|x\|)^{-n}, \quad u, v \in W_{0}, x \in X^{+}\right.
$$

But, from 14] (2.27), one deduces the existence of $r_{0} \geq 0$ such that:

$$
\mathcal{N}(x)-r_{0} \leq \mathcal{N}(u x v) \leq \mathcal{N}(x)+r_{0}, u, v \in W_{0}, x \in X^{+}
$$

But one has : $\mathcal{N}(x)=\left\langle x, 2 \rho^{\vee}\right\rangle+\left\|x^{0}\right\|, \quad x \in X^{+}$where $x^{0}$ is the projection of $x \in X \otimes \mathbb{R}$ on $Z_{X} \otimes \mathbb{R}$ along $\mathbb{Z} R_{0} \otimes \mathbb{R}$. Let us define

$$
\|v\|^{\prime}=\sup _{u \in W_{0}}\left|v\left(2 u \rho^{\vee}\right)\right|+\left\|v^{0}\right\|, \quad v \in X \otimes \mathbb{R}
$$

Then $\|\cdot\|^{\prime}$ is a norm on $X \otimes \mathbb{R}$, which is equivalent to $\|\cdot\|$. Moreover

$$
\mathcal{N}(x)=\|x\|^{\prime}, \quad \text { for all } x \in X^{+} .
$$

Hence there exists $C^{\prime \prime}>0$ such that:

$$
C^{\prime \prime-1} \mathcal{N}(x) \leq\|x\| \leq C^{\prime \prime} \mathcal{N}(x)
$$

Taking into account (6.24), (6.25) and (6.26), one gets the result.

End of the proof of the Main Theorem:

By Corollary 6.3, the image of $\mathcal{F}_{\mathcal{S}}$ is contained in the space of smooth $\mathcal{W}$-equivariant sections $C^{\infty}\left(\Xi_{u}, \operatorname{End}\left(\mathcal{V}_{\Xi}\right)\right)^{\mathcal{W}}$, and $\mathcal{F}_{\mathcal{S}}$ is continuous.

Corollary 6.10 states that the image of $\mathcal{J}_{\mathcal{C}}$ is contained in $\mathcal{S}$, and that $\mathcal{J}_{\mathcal{C}}: \mathcal{C}\left(\Xi_{u}, \operatorname{End}\left(\mathcal{V}_{\Xi}\right)\right) \rightarrow \mathcal{S}$ is continuous.

Since $C^{\infty}\left(\Xi_{u}, \operatorname{End}\left(\mathcal{V}_{\Xi}\right)\right)^{\mathcal{W}} \subset \mathcal{C}\left(\Xi_{u}, \operatorname{End}\left(\mathcal{V}_{\Xi}\right)\right)$ (see Lemma 4.2) and $\mathcal{S} \subset L_{2}(\mathcal{H})$ (see (2.14)), Corollary 3.7 implies that $\mathcal{J}_{\mathcal{C}} \mathcal{F}_{\mathcal{S}}=\mathrm{id}_{\mathcal{S}}$. It follows that the map $\mathcal{J}_{\mathcal{C}}$ in (4.5) is surjective, and that $\mathcal{F}_{\mathcal{S}}$ is injective.

Since $\mathcal{C} \subset L_{2}\left(\Xi_{u}\right.$, End $\left.\left(\mathcal{V}_{\Xi}\right), \mu_{P l}\right)$ (see Lemma 4.2), Corollary 3.7 also implies that $\mathcal{F}_{\mathcal{S}} \mathcal{J}_{\mathcal{C}}=p_{\mathcal{W}, \mathcal{C}}$. It follows, since $p_{\mathcal{W}, \mathcal{C}}$ is the identity on $C^{\infty}\left(\Xi_{u}, \operatorname{End}\left(\mathcal{V}_{\Xi}\right)\right)^{\mathcal{W}} \subset \mathcal{C}\left(\Xi_{u}, \operatorname{End}\left(\mathcal{V}_{\Xi}\right)\right)$, that $\mathcal{F}_{\mathcal{S}}$ is also surjective in (4.4). This finishes the proof of the Main Theorem. 


\section{Appendix: Some applications of spectral projec- tions}

The following lemma was suggested by Lemma 20.1 from [1] and its proof.

Lemma 7.1. Let $V$ be a complex normed vector space of dimension $p$. There exists $C>0$ such that for all $A \in \operatorname{End}(V)$ with eigenvalues less or equal to 1:

$$
\left\|A^{n}\right\| \leq C(1+\|A\|)^{p-1}(1+n)^{p}, n \in \mathbb{Z}_{+}
$$

Here $\|A\|$ is the operator norm of $A$.

Proof. Let $D_{n}$ be the disk of center 0 and radius $1+(1+n)^{-1}$. Then

$$
A^{n}=1 / 2 i \pi \int_{\partial D_{n}} z^{n}(z \operatorname{Id}-A)^{-1} d z
$$

From the Cramer rules, there exists a polynomial function from $\operatorname{End}(V)$ into itself, $B \mapsto M(B)$, of degree $p-1$, such that for any invertible $B$, one has:

$$
B^{-1}=(\operatorname{det}(B))^{-1} M(B)
$$

Hence, there exists $C^{\prime}>0$ such that:

$$
\|M(B)\| \leq C^{\prime}(1+\|B\|)^{p-1}, B \in \operatorname{End}(V) .
$$

Hence, taking into account:

$1+\|z \mathrm{Id}-A\| \leq 2+(1+n)^{-1}+\|A\| \leq\left(2+(1+n)^{-1}\right)(1+\|A\|), z \in D_{n}$, one has

$$
\|M(z \operatorname{Id}-A)\| \leq C^{\prime}\left(2+(1+n)^{-1}\right)^{p-1}(1+\|A\|)^{p-1}, z \in D_{n}
$$

Now the eigenvalues of $z \mathrm{Id}-A, z \in \partial D_{n}$ are of modulus greater or equal to $(1+n)^{-1}$. Hence

$$
|\operatorname{det}(z \operatorname{Id}-A)| \geq(1+n)^{-p}, n \in \mathbb{Z}_{+}
$$

The length of $\partial D_{n}$ is $2 \pi\left(1+(1+n)^{-1}\right)$. From equations (6.1) to (6.4), one gets:

$$
\left\|A^{n}\right\| \leq\left(1+(1+n)^{-1}\right)^{1+n} C^{\prime}\left(2+(1+n)^{-1}\right)^{p-1}(1+n)^{p}(1+\|A\|)^{p-1}
$$

One gets the required estimate with:

$$
C=C^{\prime} e 3^{p-1}
$$


Corollary 7.2. $\quad$ (i) Let $r^{\prime}>r>0$. There exists $C_{r, r^{\prime}}$ such that for all $A \in \operatorname{End}(V)$ with eigenvalues of modulus less or equal to $r$, one has:

$$
\left\|A^{n}\right\| \leq C_{r, r^{\prime}}\left(r^{\prime}\right)^{n}(1+\|A\|)^{p-1}
$$

(ii) Let $\epsilon>0$ and let $\Omega_{\epsilon}$ be the set of elements in $\operatorname{End}(V)$ whose eigenvalues are either of modulus one or of modulus less or equal to $1-\epsilon$. Let $P_{<1}$ be the sum of the spectral projections corresponding to the eigenvalues of modulus strictly less than 1. Then $P_{<1} A^{n}=\left(A_{<1}\right)^{n}$, where $A_{<1}=P_{<1} A$. Let $b>0$ such that $1-\epsilon<e^{-b}$. There exists $C$ depending on $b, \epsilon$ and $V$ such that:

$$
\left\|P_{<1} A^{n}\right\| \leq C\left(1+\left\|P_{<1} A\right\|\right)^{p-1} e^{-b n}, n \in \mathbb{N}, A \in \Omega_{\epsilon}
$$

(iii) If $A(t)$ is a continuous ( resp. holomorphic) function with values in $\Omega_{\epsilon}$, then $A_{<1}(t)$ has the same property

Proof. (i) One applies the previous result to $r^{\prime \prime-1} A$, where $r<r^{\prime \prime}<r^{\prime}$ and one uses the fact that $(1+n)^{p}\left(r^{\prime} / r^{\prime \prime}\right)^{-n}$ is bounded.

(ii) follows from (i) applied to $A_{<1}, r=1-\epsilon, r^{\prime}=e^{-b}$.

(iii) follows from the formula

$$
A_{<1}=1 / 2 i \pi \int_{\partial D} z(z \operatorname{Id}-A)^{-1} d z
$$

where $D$ is the disc of center 0 and radius $1-\epsilon / 2$.

Lemma 7.3. Let $\epsilon, a>0, p, l \in \mathbb{N}$. Let $V$ a normed complex vector space of dimension $p$ and $X=\mathbb{Z}^{l}$. Let $\pi$ be a finite dimensional complex representation of $X$. One denotes by $\left(e_{1}, \ldots, e_{p}\right)$ the canonical basis of $X$. One sets $A_{1}=\pi\left(e_{1}\right), \ldots, A_{l}=\pi\left(e_{l}\right)$. If $n=\left(n_{1}, \ldots, n_{l}\right) \in X$, one sets : $\|n\|=\left|n_{1}\right|+\cdots+\left|n_{l}\right|$, and $A^{n}=\pi(n)$.

Assume that the modulus of the eigenvalues of the $A_{i}$ are less or equal to one, and the eigenvalues of $A_{1}$ are either of modulus one or of modulus less or equal to $1-\epsilon$. Let us denote by $P_{>1}$ (resp. $P_{1}$ ) the sum of the spectral projections of $A_{1}$ corresponding to the eigenvalues of modulus strictly less than 1 (resp. of modulus 1).

Set $X_{a}^{+}=\left\{n \in \mathbb{Z}_{+}^{l} \mid n_{1}>a\|n\|\right\}$.

Then there exists $a^{\prime}$ and $C^{\prime}$, independent of the representation $\pi$ in $V$, such that:

$$
\left\|P_{<1} A^{n}\right\| \leq C^{\prime}\left(\prod_{i=1, \ldots, l}\left(1+\left\|A_{i}\right\|\right)^{p}\right) e^{-a^{\prime}\|n\|}, n \in X_{a}^{+}
$$


Proof. From Corollary 7.2 (i), (ii), one deduces that, for $b>0$ such that $1-\epsilon<e^{-b}$, and $b^{\prime}>0$, there exists a constant $C>0$, depending only on $\epsilon, b, b^{\prime}$ and $V$ such that:

$$
\left\|P_{<1} A^{n}\right\| \leq C^{\prime}\left(\prod_{i=1, \ldots, l}\left(1+\left\|A_{i}\right\|\right)^{p}\right) e^{-b n_{1}+b^{\prime}\left(n_{2}+\cdots+n_{l}\right)}, n \in X^{+}
$$

If $n \in X_{a}^{+}$, one has :

$$
b n_{1}-b^{\prime}\left(n_{2}+\cdots+n_{l}\right) \geq\left(a b-b^{\prime}\right)\|n\|
$$

$b$ being chosen, one takes $b^{\prime}=a b / 2$. Then the inequality of the Lemma is satisfied for $a^{\prime}=a b / 2$.

\section{Appendix: The $c$-function}

In this appendix we have collected some of the properties of the Macdonald $c$-function. These properties play a predominant role throughout this paper, and are closely related with the properties of residual cosets as discussed in [14], Appendix: residual cosets (Section 7).

The Macdonald $c$-function is defined as the following expression $c \in$ ${ }_{\mathcal{Q}} \mathcal{A}=\mathcal{Q} \otimes_{\mathbb{Z}} \mathcal{A}$ the quotient field of $\mathcal{A}$ :

$$
c:=\prod_{\alpha \in R_{0,+}} c_{\alpha}=\prod_{\alpha \in R_{1,+}} c_{\alpha}
$$

where $c_{\alpha}$ for $\alpha \in R_{1}$ is equal to

$$
c_{\alpha}:=\frac{\left(1+q_{\alpha^{\vee}}^{-1 / 2} \theta_{-\alpha / 2}\right)\left(1-q_{\alpha^{\vee}}^{-1 / 2} q_{2 \alpha^{\vee}}^{-1} \theta_{-\alpha / 2}\right)}{1-\theta_{-\alpha}} \in{ }_{\mathcal{Q}} \mathcal{A} .
$$

If $\alpha \in R_{0} \backslash R_{1}$ then we define $c_{\alpha}:=c_{2 \alpha}$.

Remark 8.1. We have thus associated a c-function $c_{\alpha}$ to each root $\alpha \in R_{n r}$, but $c_{\alpha}$ only depends on the direction of $\alpha$. This convention was used in [14, but differs from the one used in [13. If $\alpha \in R_{1}$ and $\alpha / 2 \notin R_{0}$, then the formula for $c_{\alpha}$ should be interpreted by setting $q_{2 \alpha^{\vee}}=1$, and then rewriting the numerator as $\left(1-q_{\alpha^{\vee}}^{-1} \theta_{-\alpha}\right)$. Here and below we use this convention.

We view $c$ as a rational function on $T$ via the isomorphism of $\mathcal{A}$ and the ring of regular functions on $T$ sending $\theta_{x}$ to the complex character $x$ of $T$.

Since the numerator and the denominator of $c$ both are products of irreducible factors whose zero locus is nonsingular (a coset of a codimension 1 subtorus of $T$ ), it is straightforward to define the pole order $i_{t}$ of $\left(c(t) c\left(w_{0} t\right)\right)^{-1}$ at a point $t \in T$ (see [14, Definition 3.2). 
Let $Q=Q\left(R_{0}\right)$ denote the root lattice of $R_{0}$. The following theorem is the main property of the $c$-function:

Theorem 8.2. (14, Theorem 7.10) We have: $i_{t} \leq \operatorname{rank}(Q)$ for all $t \in T$.

We define the notion of a "residual point" of $T$ (with respect to $(\mathcal{R}, q))$ as follows:

Definition 8.3. A point $t \in T$ is called residual if $i_{t}=\operatorname{rank}(X)$.

Corollary 8.4. There exist only finitely many residual points in $T$, for every root datum $\mathcal{R}$ and label function $q$ for $\mathcal{R}$, and the set of residual points is empty unless $Z_{X}=0$.

Proof. Let $n=\operatorname{rank}\left(R_{0}\right)$. From equation (8.1) it is clear that for any $k \in \mathbb{Z}$, the set $S_{k}:=\left\{t \mid i_{t}=k\right\}$ is a finite (possibly empty) union of nonempty Zariski open subsets of cosets $L$ of subtori of $T$, whose Lie algebra is an intersection of root hyperplanes $\alpha=0$ of $\mathbb{C} \otimes Y$. If $L$ is such a coset with $\operatorname{codim}(L)=d$, then $R_{L}:=\left\{\alpha \in R_{0}|\alpha|_{L}\right.$ is constant $\}$ is a parabolic subsystem of rank $d$. Moreover, the projection $t_{L}$ of $L$ onto $T_{L}$ is point with $i_{t_{L}}^{R_{L}}=k$. Applying Theorem 8.2 to $T_{L}$ with respect to $\left(\mathcal{R}_{L}, q_{L}\right)$ we obtain $k \leq d$.

Hence if $S_{n}$ is not finite, then there exists a proper parabolic root subsystem $R_{L} \subset R_{0}$ of rank $m<n$ say, such that $n \leq m$, which is clearly absurd. The remaining part of the Corollary is straightforward from Theorem 8.2 .

Remark 8.5. There is a classification of residual points (cf. [9], 14]).

Another fact of great consequence is the following.

Theorem 8.6. (14], Theorem 7.14) Let $r=s c \in T$ be residual, with $s \in T_{u}$ and $c \in T_{r s}$. Then $r^{*}:=s c^{-1} \in W\left(R_{s, 1}\right) r$, where $R_{s, 1}$ is the root subsystem of $R_{1}$ defined by $R_{s, 1}:=\left\{\alpha \in R_{1} \mid \alpha(s)=1\right\}$.

We extend the definition of the $c$-function to arbitrary standard induction data. First recall Theorem 2.8, stating that the central character of a residual discrete series representation is residual.

Definition 8.7. Let $\xi=\left(P, \delta, t^{P}\right)$ be a standard induction datum, and let $r_{P} \in T_{P}$ be such that $W_{P} r_{P}$ is the central character of $\delta$ (thus $r_{P}$ is $a\left(\mathcal{R}_{P}, T_{P}\right)$-residual point). Put $t=r_{P} t^{P} \in T$. We define:

$$
c(\xi):=\prod_{\alpha \in R_{0,+} \backslash R_{P,+}} c_{\alpha}(t)
$$

Notice that we recover the original c-function defined on $T$ as the special case where $P=\emptyset$ and $\delta=\mathbb{C}$. 
The next proposition goes back to 9], Theorem 3.13 (also see 14, Theorem 3.25).

Proposition 8.8. Let $P \subset F_{0}$ and let $\xi=\left(P, \delta, t^{P}\right) \in \Xi_{P, \delta, u}$. Choose $r_{P} \in T_{P}$ such that $W_{P} r_{P}$ is the central character of $\delta$, and let $t=$ $r_{P} t^{P} \in T$.

(i) $c\left(\xi^{-1}\right)=c\left(w^{P}(\xi)\right)=\overline{c(\xi)}$.

(ii) The function $\xi \rightarrow|c(\xi)|^{2}$ on $\Xi_{u}$ is $\mathcal{W}$-invariant.

(iii) The function $c(\xi)$ is $\mathcal{K}$-invariant.

(iv) Let $P^{\prime} \in \mathcal{P}$ and $d \in K_{P^{\prime}} \times W\left(P, P^{\prime}\right)$. The rational functions $c(d \xi) c(\xi)^{-1}$ and $c(d \xi)^{-1} c(\xi)$ (of $\xi \in \Xi_{P, \delta}$ ) are regular in a neighborhood of $\Xi_{P, \delta, u}$.

(v) The rational function $c(\xi)^{-1}$ is regular in a neighborhood of $\Xi_{u}$.

Proof. (i) A straightforward computation from the definitions, using Theorem 8.6 (cf. [14, (3.58)).

(ii) The $\mathcal{W}$-invariance follows simply from the definitions if we write (using (i)) $|c(\xi)|^{-2}=\left(c(\xi) c\left(\xi^{-1}\right)^{-1}\right.$ (cf. 14, Proposition 3.27).

(iii) This follows trivially from the definition of the action of $\mathcal{K}$ on $\xi$ : If $k \in K_{P}$ then $k \xi=k\left(P, \delta, t^{P}\right)=\left(P, \Psi_{k}(\delta), k t^{P}\right)$. The central character of $\Psi_{k}(\delta)$ is equal to $k^{-1} W_{P} r_{P}=W_{P}\left(k^{-1} r_{P}\right)$, thus we need to evaluate the $c_{\alpha}$ in the product $c(k \xi)$ at the point $k^{-1} r_{P} k t^{P}=t$, or any of its images under the action of $W_{P}$. Hence $c(k \xi)=c(\xi)$.

(iv) By (i) and (ii) it is clear that these rational functions have modulus 1 on $\Xi_{P, \delta, u}$ (outside their respective singular sets).

The singular sets of these rational functions are of the following form. Choose $r_{P} \in T_{P}$ such that $W_{P} r_{P}$ is the central character of $\delta$. Then the singular set of $c(d \xi) c(\xi)^{-1}$ is the union of the zero sets of the functions

$$
\prod_{\alpha \in R_{1,+} \backslash R_{P, 1,+}}\left(1-\alpha_{P, \delta}^{-1}\right)
$$

and

$$
\prod_{\alpha \in R_{1,+} \backslash R_{P, 1,+}}\left(1+q_{\alpha^{\vee}}^{-1 / 2} \alpha_{P, \delta}^{-1 / 2}\right)\left(1-q_{\alpha^{\vee}}^{-1 / 2} q_{2 \alpha^{\vee}}^{-1} \alpha_{P, \delta}^{-1 / 2}\right)
$$

on $\Xi_{P, \delta}$, where $\alpha_{P, \delta}$ denotes the function on $\Xi_{P, \delta}$ defined by $\alpha_{P, \delta}(\xi)=$ $\alpha\left(r_{P} t^{P}\right)$.

In the case of $c\left(w^{P} \xi\right)^{-1} c(\xi)$ the answer is the same, but we need to take the products over the set $\alpha \in d^{-1} R_{1,+} \backslash R_{P, 1,-}$.

The intersection of a component of this hypersurface with $\Xi_{P, \delta, u}$ is either empty or it has (real) codimension 1 in $\Xi_{P, \delta, u}$. 
By the boundedness of $c(d \xi) c(\xi)^{-1}$ on $\Xi_{P, \delta, u}$, this implies that the pole order of this function at a components of the singular set which meets $\Xi_{P, \delta, u}$ is in fact equal to zero. Hence the poles are removable in a neighborhood of $\Xi_{P, \delta, u}$. Similarly for $c(d \xi)^{-1} c(\xi)$.

(v) The proof of [9], Theorem 3.13 may be adapted to the present situation. Or we may argue as in (iv) as follows.

Since $|c(\xi)|^{-2}=\left(c(\xi) c\left(w^{P}(\xi)\right)\right)^{-1}$ is smooth on $\Xi_{u}$ (cf. [14, Theorem 3.25 , equation (3.53), Proposition 3.27 and equation (3.58)), it follows that $c(\xi)^{-1}$ is bounded on $\Xi_{P, \delta, u}$. Hence the argument that was used in the proof of (iv) applies.

\section{References}

[1] E.P. van den Ban, The principal series for a reductive symmetric space II, J. Funct. Analysis 109 (1992), pp. 331-441.

[2] E.P. van den Ban, J. Carmona, P. Delorme, Paquets d'onde dans l'espace de Schwartz d'un espace symétrique réductif, J. Funct. Analysis 139 (1996), pp. $225-243$.

[3] N. Bourbaki, Groupes et Algèbres de Lie, Chaps IV,V,VI, Hermann, Paris (1972).

[4] R.W. Carter, Finite groups of Lie type, Wiley Classics Library, John Wiley and sons, Chichester, UK.

[5] P. Delorme (avec un appendice de M. Tinfou), Espace de Schwartz pour la transformée de Fourier hypergéométrique, J. Funct. Anal., 168 (1999), pp. 239-312.

[6] J. Dixmier, Les $C^{*}$-algèbres et leurs representations, Cahiers scientifiques, fasc. XXIX (1969), Gauthier-Villars, Paris.

[7] Harish-Chandra, Harmonic analysis on real reductive groups II, Wave packets in the Schwartz space, Inv. Math 36 ( 1976), pp. 1-35.

[8] Harish-Chandra, Harmonic analysis on real reductive groups III, The MaassSelberg relations and the Plancherel formula, Ann. Math. 104 (1976), pp. 117201.

[9] G.J. Heckman, E.M. Opdam, Yang's system of particles and Hecke algebras, Annals of mathematics 145 (1997), pp. 139-173.

[10] A.W. Knapp, Representation of semisimple groups, An overview based on examples, Princeton Math. Series 36, Princeton University Press (1986).

[11] G. Lusztig, Affine Hecke algebras and their graded version, J. Amer. Math. Soc. 2 (1989), no. 3, pp. 599-635.

[12] H. Matsumura, Commutative Ring Theory, Cambridge studies in advanced mathematics 8, Cambridge University Press, (1986).

[13] E.M. Opdam, A generating formula for the trace of the Iwahori-Hecke algebra, Progress in Mathematics 210 (2003), pp. 301-323 (also math.RT/0101006).

[14] E.M. Opdam, On the spectral decomposition of affine Hecke algebras, JIMJ, (to appear). 


\section{Index}

$(\cdot, \cdot)$

inner product on $\mathcal{H}, 10$

inner product on $\operatorname{Pol}\left(\operatorname{End}\left(\mathcal{V}_{(\mathcal{K} \backslash \Xi)}\right)\right)$, 21

$\|\cdot\|$, norm on the vector space $\mathbb{Q} \otimes_{\mathbb{Z}}$ $Z_{X}, 6$

$\|\cdot\|_{o}$, operator norm on $\mathcal{H}, 10,24$

$h \rightarrow h^{*}$, conjugate linear anti-involution of $\mathcal{H}, 10$

$\langle\cdot, \cdot\rangle$

perfect pairing between $X$ and $Y$, 5

inner product on $V_{\pi}, 17$

$\leq_{P}$, partial ordering of exponents relative to $P \in \mathcal{P}, 14,25$

$\Delta=\Delta_{\mathcal{R}, q}$, complete set of representatives of the irreducible discrete series representations of $\mathcal{H}(\mathcal{R}, q)$, 10

$\Delta_{W_{0} r}$, subset of $\Delta$ consisting of the representations with central character $W_{0} r, 10$

$\Delta_{P}=\Delta_{\mathcal{R}_{P}, q_{P}}$, complete set of representatives for the equivalence classes of irreducible discrete series representations of $\mathcal{H}_{P}$ $=\mathcal{H}\left(\mathcal{R}_{P}, q_{P}\right), 18$

$\Xi$ set of standard induction data $\xi=$ $\left(P, \delta, t^{P}\right)$ with $P \in \mathcal{P}, \delta \in \Delta_{P}$ and $t^{P} \in T^{P}, 17$

$\Xi_{P}$, set of standard induction data of the form $\left(P, \delta, t^{P}\right)$ with $\delta \in \Delta_{P}$ and $t^{P} \in T^{P}, 20$

$\Xi_{u}$ set of tempered standard induction data $\xi=\left(P, \delta, t^{P}\right), 17$

$\Psi_{g}: \Delta_{P} \rightarrow \Delta_{Q}$, bijection induced by $\psi_{g}, 18$

$\Omega$, length 0 elements in $W, 5$

$\Omega_{f}=\Omega / Z_{X}, 6$

$\tilde{\delta}_{g}: V_{\delta} \rightarrow V_{\Psi_{g}(\delta)}$, unitary isomorphism intertwining the representations $\delta \circ \psi_{g}^{-1}$ and $\Psi_{g}(\delta), 19$

$\theta_{x}$, basis elements of $\mathcal{A}, 8$

$\iota_{s}$, intertwining element of $\mathcal{H}, 9$ $\iota_{s}^{0}$, normalized intertwining element of ${ }_{\mathcal{Q}}^{\mathcal{H}}, 9$

$\mu_{P l}$, Plancherel measure, spectral measure of the tracial state $\tau$ on $C_{r}^{*}(\mathcal{H})$, 21

$\nu$, (multiplicative) distance function on $T_{r s}, 35$

$\xi=\left(P, \delta, t^{P}\right)$ standard induction datum, 17

$\pi$, induction functor on $\mathcal{W}_{\Xi}, 20$

$\pi\left(\mathcal{R}_{P}, W_{P} r, \delta, t\right)=\operatorname{Ind}_{\mathcal{H}^{P}}^{\mathcal{H}}\left(\delta_{t}\right)$, parabolically induced representation, 17

$\pi(\xi):=\pi\left(\mathcal{R}_{P}, W_{P} r_{P}, \delta, t^{P}\right)$, where $\xi=$ $\left(P, \delta, t^{P}\right)$ and $W_{P} r_{P}$ is the central character of $\delta, 18$

$\varpi$, equivalence class inside $W_{0} t, 15$

$\pi(g, \xi)$, unitary intertwining operator, intertwining $\pi(\xi)$ and $\pi(g \xi), 18$

$\tau$, trace functional of $\mathcal{H}, 10$

$\phi_{t^{P}}: \mathcal{H}^{P} \rightarrow \mathcal{H}_{P}$, family of surjective homomorphisms, 9

$\psi_{g}: \mathcal{H}^{P} \rightarrow \mathcal{H}^{Q}$, isomorphism for $g \in$ $K_{Q} \times W(P, Q), 18$

$\mathcal{A}$, abelian subalgebra of $\mathcal{H}, 8$

$\mathbb{A}$, space of finite functionals on $\mathcal{H}, 12$ $\mathbb{A}^{\text {cusp }}$, cuspidal elements in $\mathbb{A}^{\text {temp }}, 14$

$\mathbb{A}^{\text {temp }}$, tempered elements in $\mathbb{A}, 14$

${ }_{\mathcal{Q}} \mathcal{A}=\mathcal{Q} \otimes_{\mathbb{Z}} \mathcal{A}$, quotient field of $\mathcal{A}, 45$

$a=\left(\alpha^{\vee}, k\right)$, affine root, 6

$C^{\infty}\left(\Xi_{u}\right)$, space of $C^{\infty}$-functions on $\Xi_{u}, 37$

$C^{\infty}\left(\Xi_{u}, \operatorname{End}\left(\mathcal{V}_{\Xi}\right)\right)$, space of smooth sections in $\operatorname{End}\left(\mathcal{V}_{\Xi}\right), 22$

$C_{r}^{*}(\mathcal{H})$, the reduced $C^{*}$ algebra of $\mathcal{H}$, 10

$\mathcal{C}\left(\Xi_{u}, \operatorname{End}\left(V_{\Xi}\right)\right)=c C^{\infty}\left(\Xi_{u}, \operatorname{End}\left(V_{\Xi}\right)\right)$, 23

$c(\xi)=\prod_{\alpha \in R_{0,+} \backslash R_{P,+}} c_{\alpha}(t)$, Macdonald's $c$-function on $\Xi$, where $\xi=$ $\left(P, \delta, t^{P}\right)$, and $t=r_{P} t^{P}$ with $W_{P} r_{P}$ the central character of $\delta, 45$

$c_{\alpha}$, rank one $c$-function, 45

central character of $(V, \pi), 8$ 
$e_{\varpi}$, idempotent of $\overline{\mathcal{H}}_{t}$ associated with $\varpi \subset W_{0} t, 15$

$F^{\text {aff }}$, affine simple roots, 5

$F_{0} \subset R_{0}$, simple roots of $R_{0}, 5$

$\mathcal{F}$, Fourier Transform, 21

$\mathcal{F}_{\mathcal{H}}, \mathcal{F}$ restricted to $\mathcal{H}, 21$

$\mathcal{F}_{\mathcal{S}}, \mathcal{F}$ restriced to $\mathcal{S}, 23$

$\mathcal{F}_{C}, \mathcal{F}$ restricted to $C_{r}^{*}(\mathcal{H}), 24$

$f_{s}=\log _{\mathbf{q}}(q(s)), 7$

$\mathcal{H}$, affine Hecke algebra, 7

${ }_{\mathcal{Q}} \mathcal{H}=\mathcal{Q} \otimes_{\mathcal{Z}} \mathcal{H}, 9$

$\mathcal{H}^{*}$, algebraic dual of $\mathcal{H}, 12$

$\mathcal{H}_{0}=\mathcal{H}\left(W_{0},\left.q\right|_{S_{0}}\right) \subset \mathcal{H}, 8$

$\mathcal{H}^{P}=\mathcal{H}\left(\mathcal{R}^{P}, q^{P}\right)$, parabolic subalgebra of $\mathcal{H}, 8$

$\mathcal{H}_{P}=\mathcal{H}\left(\mathcal{R}_{P}, q_{P}\right)$, semisimple quotient of $\mathcal{H}^{P}, 8$

$\overline{\mathcal{H}}_{t}=\overline{\mathcal{Z}}_{W_{0} t} \otimes_{\mathcal{Z}} \mathcal{H}, 14$

$\mathcal{H}^{t}=\mathcal{H} / \mathcal{I}_{t} \mathcal{H}$, where $\mathcal{I}_{t}$ is the maximal ideal of $W_{0} t$ in $\mathcal{Z}, 16$

$\overline{\mathcal{H}^{t}}$, the quotient of $\mathcal{H}^{t}$ by the radical of the positive semi-definite Hermitian pairing $(x, y)_{t}:=\chi_{t}\left(x^{*} y\right)$, 27

$i\left(V_{\delta}\right)=\mathcal{H}\left(W^{P}\right) \otimes V_{\delta}$, underlying vector space of $\pi\left(P, \delta, t^{P}\right)$ in the compact realization, 17

$i_{t}$, pole order of $\left(c(t) c\left(w_{0} t\right)\right)^{-1}$ at $t \in$ $T, 45$

$\mathcal{J}$, wave packet operator, adjoint of $\mathcal{F}, 22$

$\mathcal{J}_{\mathcal{C}}$, restriction of $\mathcal{J}$ to the Fréchet space $\mathcal{C}\left(\Xi_{u}, \operatorname{End}\left(\mathcal{V}_{\Xi}\right)\right), 23$

$K_{P}$, finite abelian group $T_{P} \cap T^{P}, 18$

$\mathcal{K} \triangleleft \mathcal{W}$, normal subgroupoid whose set of objects is $\mathcal{P}$, with $\operatorname{Hom}_{\mathcal{K}}(P, Q)$ $=K_{P}$ if $P=Q$ and $\emptyset$ else, 21

$L_{2}(\mathcal{H})$, Hilbert completion of $\mathcal{H}, 10$ $l$, length function on $W, 5$

$\mathbb{N}=\{1,2,3, \cdots\}, 5$

$N_{w}$, basis elements of $\mathcal{H}, 7$

$\mathcal{N}$, norm function on $W, 6$

$P \in \mathcal{P}$, subset of $F_{0}, 6$
$\mathcal{P}$, power set of $F_{0}, 6$

$p_{\mathcal{W}}$, averaging operator for the action of $\mathcal{W}$ on sections of $\operatorname{End}\left(\mathcal{V}_{\Xi}\right), 20$

$\operatorname{Pol}\left(\operatorname{End}\left(\mathcal{V}_{\Xi}\right)\right)^{\mathcal{W}}$, space of $\mathcal{W}_{\Xi}$-equivariant sections in $\operatorname{Pol}\left(\operatorname{End}\left(\mathcal{V}_{\Xi}\right)\right), 20$

$\operatorname{Pol}(\Xi)$, space of Laurent polynomials on $\Xi, 20$

$Q$

$Q=Q\left(R_{0}\right)$, root lattice, 5

$Q \in \mathcal{P}$, subset of $F_{0}, 6$

$\mathcal{Q}$, quotient field of $\mathcal{Z}, 9$

q, base for the labels $q(s), 7$

$q, l$-multiplicative function on $W, 6$

$q^{P}$, label function on $\mathcal{R}^{P}$ determined by the restriction of $q$ to $R_{P, \mathrm{nr}}, 7$

$q_{P}$, label function on $\mathcal{R}_{P}$ determined by the restriction of $q$ to $R_{P, \mathrm{nr}}, 7$

$q_{a}$, affine root label, 6

$q_{\alpha^{\vee}}$, label for $\alpha^{\vee} \in R_{\mathrm{nr}}^{\vee}, 7$

$\mathcal{R}$, root datum, 5

$\mathcal{R}^{P}$, root datum associated to $P, 6$

$\mathcal{R}_{P}$, semisimple root datum associated to $P, 6$

$R_{0} \subset X$, reduced integral root system, 5

$R_{0}^{\vee} \subset Y$, coroot system, 5

$R_{P} \subset R_{0}$, parabolic subsystem, root system of $\mathcal{R}_{P}, 6$

$R_{1}$, system of long roots in $R_{\mathrm{nr}}, 7$

$R_{\mathrm{nr}}$, non reduced root system, 7

$R^{\text {aff }}$, affine root system, 5

$R_{ \pm}^{\text {aff }}$, positive (negative) affine roots, 5

$\operatorname{Rat}^{r e g}(\Xi)$, rational functions on $\Xi$, regular in an open neighborhood of $\Xi_{u} \subset \Xi, 20$

$S^{\text {aff }}$, simple reflections of $W^{\text {aff }}, 5$

$S_{0}$, simple reflections of $W_{0}, 5$

$\mathcal{S}$, the Schwartz completion of $\mathcal{H}, 11$

$s_{\alpha}$, reflection in $\alpha, 16$

$T=\operatorname{Hom}_{\mathbb{Z}}\left(X, \mathbb{C}^{\times}\right)$, complex algebraic torus, 8

$T^{P} \subset T$, algebraic subtorus of $T$ with $\operatorname{Lie}\left(T^{P}\right)=P^{\perp}, 16$ 
$T_{P} \subset T$, algebraic subtorus orthogonal to $T^{P}$, with character lattice $X_{P}, 17$

$T_{r s}=\operatorname{Hom}\left(X, \mathbb{R}_{+}^{\times}\right)$, real split form of $T, 11$

$T_{u}=\operatorname{Hom}\left(X, S^{1}\right)$, compact form of $T, 11$

$|t| \in T_{r s}$, real split part of $t \in T, 11$

$V_{\xi}=i\left(V_{\delta}\right)$, underlying vector space of $\pi(\xi), 20$

$\mathcal{V}_{\Xi}$, trivial fiber bundle over $\Xi, 20$

$W$, affine Weyl group, 5

$W_{\sigma}$, wave packet, 39

$W_{0}$, Weyl group of $R_{0}, 5$

$W^{\text {aff }}=W_{0} \ltimes Q \subset W, 5$

$W_{P}$, Weyl group of $R_{P}$, parabolic subgroup $W_{0}, 6$

$W^{P}=W_{0} / W_{P}$, set of left cosets $w W_{P}$. If $P \subset F_{0}$, identified with shortest length representatives, 6

$W(P, Q)=\left\{w \in W_{0} \mid w(P)=Q\right\}$, with $P, Q \in \mathcal{P}, 18$

$\mathcal{W}$, groupoid whose set of objects is $\mathcal{P}$, with morphisms $\operatorname{Hom}_{\mathcal{W}}(P, Q)=$ $\mathcal{W}(P, Q):=K_{Q} \times W(P, Q), 18$

$\mathcal{W}_{\Xi}:=\mathcal{W} \times \mathcal{P} \Xi$, groupoid of standard induction data, 18

$\mathcal{W}_{P}$, set of elements of $\mathcal{W}$ with source $P \in \mathcal{P}, 20$

$\mathcal{W}_{\xi}=\mathcal{W}_{P}$ if $\xi \in \Xi_{P}, 20$

$\mathcal{W}_{\Xi_{u}}:=\mathcal{W} \times_{\mathcal{P}} \Xi$, groupoid of tempered standard induction data, 18

$w_{0}$, longest element of $W_{0}, 31$

$w_{P}$, longest element of $W_{P}, 31$

$w^{P}$, longest element of $W^{P}, 31$

$X, Y$, lattices, 5

$X^{+}$, cone of dominant elements in $X$, 6

$X_{P} \supset R_{P}$, lattice of $\mathcal{R}_{P}$, character lattice of $T_{P}, 6$

$Y_{P} \supset R_{P}^{\vee}$, lattice of $\mathcal{R}_{P}$, cocharacter lattice of $T_{P}, 6$

$\mathbb{Z}_{+}=\{0,1,2, \ldots\}, 5$

$Z_{X}$, length 0 translations in $W, 6$

$\mathcal{Z}$, the center of $\mathcal{H}, 8$
$\overline{\mathcal{Z}}_{W_{0} t}$, formal completion of $\mathcal{Z}$ at $W_{0} t$, 14 
Institut de Mathématiques de Luminy, UPR 9016 Du CNRS, Faculté des Sciences de Luminy, 163 Avenue de Luminy, Case 901, 13288 MarSeille Cedex 09, France, email: Delorme@iml.univ-Mrs.Fr

Korteweg de Vries Institute for Mathematics, University of Amsterdam, Plantage Muidergracht 24, 1018TV Amsterdam, The NetherLANDS, EMAIL: OPDAM@SCIENCE.UVA.NL 